\title{
Information-Traffic Coupled Cell Transmission Model for Information Spreading Dynamics over Vehicular Ad Hoc Network on Road Segments
}

\author{
Lili $\mathrm{Du}^{1}$, Siyuan Gong ${ }^{2}$, Lu Wang ${ }^{3}$, Xiang-Yang $\mathrm{Li}^{4}$
}

\begin{abstract}
Vehicular Ad Hoc Network (VANET) makes real-time traffic information accessible to vehicles en routes, thus possesses a great potential to improve traffic safety and mobility in the near future. Existing literature shows that we are still lack of approaches to track information spreading dynamics via VANET, which will prevent the potential applications from success. Motivated by this view, this research develops an information-traffic coupled cell transmission model (IT-CTM) to capture information spreading dynamics via VANET. More exactly, this study considers information spreading over a road segment forms a wave with a front and tail, each of which goes through the road segment following an intermittent transmission pattern due to traffic flow dynamics. The approach of IT-CTM discretizes a road segment into a number of cells. Each cell covers several intermittent transmissions. Mathematical methods are developed to capture the inner-cell and inter-cell movements of information front and tail, which enable us to track the information spreading dynamics along cells. Numerical experiments based on simulation and field data indicate that the IT-CTM can closely track the dynamic movements of information front and tail as well as the dynamic information coverage as a single or multiple piece(s) of information propagating via VANET on a one-way or two-way road segment. The mean absolute error (MAE) for tracking dynamic information coverage is less than 5\% across all experiments in this study.
\end{abstract}

Key words: VANET; cell transmission model; information spreading; coverage; dynamics

\footnotetext{
${ }^{1}$ Corresponding author, Department of Civil, Architectural and Environmental Engineering, Illinois Institute of Technology, 3201 South Dearborn Street, Chicago, Illinois 60616 U.S.A., Email: 1du3@iit.edu

${ }^{2}$ Department of Civil, Architectural and Environmental Engineering, Illinois Institute of Technology, 3201 South Dearborn Street, Chicago, Illinois 60616 U.S.A., Email: sgong1 @ hawk.iit.edu

${ }^{3}$ Department of Civil, Architectural and Environmental Engineering, Illinois Institute of Technology, 3201 South Dearborn Street, Chicago, Illinois 60616 U.S.A., Email: 1wang121 @ hawk.iit.edu

${ }^{4}$ Department of Computer Science, Illinois Institute of Technology, 10 West 31st Street, Chicago, IL, 60616, Email: xli@cs.iit.edu
} 


\section{Introduction}

Vehicular Ad Hoc Network (VANET) (also named as Vehicle-to-Vehicle (V2V) communication network or inter-vehicle communication network in the literature), enabling information exchange among vehicles through $5.9 \mathrm{GHz}$ DSRC wireless communication (FCC, 1999), is a well-known technology, which possesses a great potential to improve traffic safety (Bai and Krishnan 2006), mobility (Bauza et al., 2010) and environmental sustainability (Alsabaan et al., 2010; Tielert et al., 2010). The promising applications include real-time collision alerts, hazardous warning, coordinated driving, real-time navigation, traffic accident information warning, etc. Along with the development of these applications, it has been recognized that information availability plays a critical role to achieve the promise. For example, the application of real-time forward collision warning needs to know how fast information can reach target vehicles; spreading accident warning through VANET needs to have the knowledge about the information coverage in a local transportation network before the information expires. Accordingly, extensive research has been motivated to investigate information propagation in VANET from different perspectives. While wireless communication community mainly focused on designing communication protocols (Biswas et al., 2006; Dar et al., 2008), privacy protection (Burmester et al., 2008), safety (Nekovee, M. 2006), routing protocols (Chen et al., 2009; Taleb et al., 2007), etc, transportation community seeks to capture information propagation characteristics (such as connectivity, transmission distance, time delay, coverage) in VANET associated with traffic stream features. This study shares the common interests of the latter, thus mainly reviews previous research in transportation community. Along with the review, we also differentiate and highlight the main contributions of this study.

Frist of all, many studies have explored instantaneous information propagation in VANET. The investigated topics include communication opportunity, instantaneous propagation distance, speed, and connectivity. The representative literatures are briefly discussed below. Jin and Recker (2006) computed the probability of a successful instantaneous information transmission between two vehicles in uniform and general traffic streams. Both Ukkusuri and Du (2008) and Jin and Recker (2010) developed analytical formulations to estimate the multi-hop connectivity of inter-vehicle communication network assuming stationary traffic stream, but different mathematical models are used. Wang (2007) developed mathematical formulas to provide the mean and variance of instantaneous information propagation distance as well as its distribution in VANET, considering the presence of equipped vehicles follows an independent homogeneous Poisson process. Wang et al. (2010) revisited this problem and made their approach adaptive to vehicle headway following a general distribution. Furthermore, Wang et al. (2012) and Yin et al. (2013) developed mathematical methods to estimate the expectation, variance, and probability distribution of instantaneous information propagation distance on parallel roads, assuming that vehicles' headway follows Gamma, Poisson, or Log-normal distribution. Clearly, this group of research studies information propagation at a snapshot under static traffic flow, since information propagation via wireless communication is much faster (in order of millisecond) than traffic flow variation (in order of minutes). Accordingly, the effect of traffic dynamics on information spreading is not fully considered.

Other literatures studied intermittent information propagation via VANET. Namely, information alternatively propagates through wireless communication or by vehicle carrying as wireless communication is not available. In what follows, we discuss some representative work. Artimy et al. (2005a, 2005b, 2006) examined the connectivity of VANET with static or dynamic assigned transmission range considering traffic flow dynamics. Schönhof et al. (2006) investigated how smart vehicle density impacts information propagation speed and efficiency considering dynamic communication link in a dynamic traffic flow on a two-way freeway traffic stream. Agarwal et al. (2008) studied the delay tolerant message propagation in VANET and developed the upper and lower bounds for information propagation speed as the functions of traffic density, vehicle speed, and transmission range. Wu et al. (2009) indicated that information propagation distance and speed depend on relative vehicle movement and other traffic characteristics such as vehicle density and average vehicle speed; Wang et al. (2014) proposed an analytical model to estimate the expected information propagation speed in a traffic flow with low smart vehicle penetration. Both instantaneous and carrier transmissions are considered. Du and Ukkusuri (2010) 
modeled intermittent information connectivity over a VANET on a one-way road segment by a timeexpanded network, and then provided a closed-form formulation to estimate the average network connectivity (reachability) over a time period. Du and Dao (2015) provided the closed-form formulations to estimate the expected time delay by which a piece of information goes through a one-way or two-way road segments following intermittent information propagation via VANET. Kim et al. (2015) proposed an integrated model consisting of integro-differential equations to describe the information flow propagation mechanism and a partial differential equation to describe the traffic flow dynamics. The proposed approach provides a closed-form solution for the speed of the information flow propagation front. Overall, this group of research has made great contributions to understand the information propagation speed, distance, and time delay via VANET, considering various traffic conditions and necessary communication constraints. However, few existing solutions are able to track the information spreading dynamics via a VANET, while this is the main focus of the proposed study.

A few recent studies considered the information coverage via VANET over a local network. For example, by utilizing the information propagation model proposed in Wang (2007), $\mathrm{Ng}$ and Waller (2010) provided the lower and upper bounds of information propagation delay between two nodes in a network, where traffic flow characteristics are evaluated by a static traffic assignment model. Osman and Ishakv (2015) developed a new measure of effectiveness to capture the connectivity robustness in a connected vehicle environment that accounts for traffic density, transmission range, and market penetration, which affect the robustness and stability of a connected vehicle environment.

The brief review above indicates two new research needs. First, even though existing solutions in literature provided various approximation methods to characterize information propagation, very few studies investigated the dynamic information spreading process via VANET, which is one of the most critical and unique features for VANET. Second, existing studies mainly considered a single piece of information, but in reality multiple pieces of information will propagate in the network simultaneously. We are still lack of the knowledge about the information merging or aggregation. This study seeks to partially make up the above gap.

The proposed approach considers that all vehicles on a road segment are equipped with wireless communication facilities; a piece of information, once initiated, flow through the road segment via a VANET formed by vehicles on this road. The spreading of a piece of information forms an information wave with a front and a tail. The movements of the information front and tail, which present the spreading of the information, follow an intermittent transmission pattern, which is significantly impacted by traffic flow dynamics. Specifically, the information front or tail may smoothly jump from one vehicle to another as two vehicles are well connected through wireless communication (i.e. instantaneous transmission), or they can also possibly get stuck at a vehicle and move with the vehicle (i.e. ferry transmission) as no wireless connection available around. Built upon this view, this research develops an information and traffic coupled cell transmission model (IT-CTM) to capture information spreading dynamics based upon traffic flow dynamics, employing the similar idea of CTM developed by (Daganzo, 1994).

The proposed IT-CTM involves the following innovative approaches. (i) IT-CTM determines cell length considering both traffic flow and information flow propagation characteristics. (ii) IT-CTM captures the information spreading dynamic process by tracking the movement of the information front and tail along a chain of cells. Accordingly, descriptive models and regulations are developed to formulate inner-cell and inter-cell information propagation, considering homogeneous and nonhomogeneous traffic flow respectively. Here, existing efforts (such as Du and Dao (2015), which provide closed form formulations to calculate information propagation distance, speed, or delay under instantaneous or ferry transmissions, can be integrated into the IT-CTM, if those formulations employed macroscopic vehicle distribution information. (iii) IT-CTM is able to capture the merging of information spreading as multiple pieces of information land on a road segment sequentially. (iv) The numerical experiments demonstrate that the IT-CTM can closely track information spreading dynamics over spatialtemporal dimensions with the average mean absolute error less than 5\% under various traffic conditions.

To the best of our knowledge, this is the first effort to explore the information spreading dynamics seamlessly integrating traffic flow dynamics. Existing statistical results about information propagation 
characteristics (distance, speed, time delay, etc) under instantaneous or ferry transmissions can be also integrated into the proposed IT-CTM. Thus, this research will initiate a new line of research and significantly contribute to this research field in literature. Moreover, the proposed approach has a great potential to be integrated into current macroscopic traffic simulators and extend its functionality to track information propagation dynamics. Again to our best knowledge, there is not such well-developed simulator existing in literature. From the application point of view, it has been well accepted that the success of most applications built upon VANETs (e.g. online traffic accidents alarm) relies upon timely and sufficient information provision and updates. The proposed IT-CTM approach will help us better understand the information availability which will significantly sustain their successful implementation in practice.

The work of this paper is structured as below. Section 1 introduces the research background and motivations. Section 2 describes the road segment and traffic flow, and further formulates information flow and the intermittent information movement. Section 3 proposes information-traffic coupled cell transmission model as well as the approach to capture information spreading dynamics over traffic flow dynamics on a road segment. The proposed approaches are validated by the numerical experiments presented in Section 4. We conclude this research in Section 5.

\section{Preliminaries}

This section describes the road segment of interests and intermittent information flow transmission model. The important concepts are introduced, including intermittent transmission pattern, information wave front, tail, and forward and backward directions for information propagation. Based on that, we introduce the formulations to calculate the movement distance of an intermittent transmission.

\subsection{Information wave front, tail and direction}

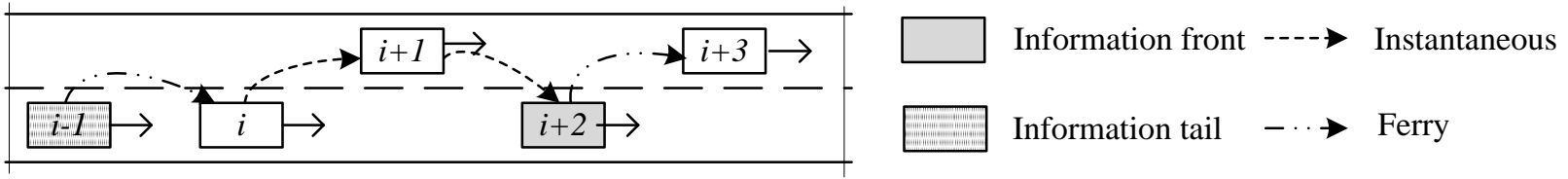

Figure 1 Information front and tail

This study considers information propagate via a VANET using broadcast protocol along a road segment of the length $L$ only with two ends: one is traffic flow entrance and the other is exit. Traffic flow composed of vehicles all equipped with wireless communication facilities, moves on a one-way or twoway road segment with multiple lanes. Two vehicles will build up successful wireless communication through V2V communication, if the distance between two vehicles is less than a transmission range $r$ (Gupta and Kumar, 2000; Du and Dao, 2015). Even though an information transmitter (i.e., a vehicle broadcasting information) may reach multiple receivers (i.e. vehicles in the transmission range) in the transmission range, this study only considers the nearest receiver within the transmission range for the same reasons given in Section III.B by (Du and Dao, 2015).

A piece of information may land on a road segment from its traffic flow entrance or exit. The spreading of a piece of information on the road segment forms two boundaries, i.e., one is front and the other is tail. All the vehicles between the front and tail are considered as informed vehicles. Without loss of generality, this study considers that the end of the road segment where a piece of information first lands on as the origin, from which an information spreading wave starts. Accordingly, an information front is always represented by an informed vehicle furthest away from the origin, while an information tail is always represented by an informed vehicle closest to the origin. Figure 1 illustrates an example, in which information first lands on from the left side end, and then vehicle $i+2$ represents the front and vehicle $i-1$ represents the tail.

According to the definitions of information front and tail, this study always considers the direction from an information tail to front as the forward information wave spreading direction, and then the 
opposite direction as the backward direction. For the given example in Figure 1, the direction from vehicle $i-1$ to vehicle $i+2$ represents the forward information spreading direction, which is in eastbound (hereafter, the eastbound and westbound are denoted as EB and WB respectively). Both information front and tail will propagate backward or forward along traffic flow and may leave the road segment after a number of transmissions. Section 3 studies the movement of information front and tail to describe information spreading dynamics.

Clearly, a piece of information may spread along traffic flow. Accordingly, an information front or tail may move forward or backward along or opposite to traffic flow. With a pre-defined information spreading direction and traffic flow direction, Figure 2 illustrates four possible cases to describe the relationship between traffic flow and information wave. Cases (a) and (b) in Figure 2, respectively, illustrate that the information front and tail moves along or opposite to traffic flow direction on a one-way road. Case (c) and Case (d) describe the propagation of information front/tail on a two-way traffic road segment. It is noticed that Case (c) and Case (d) are essentially the same, since the movements of information front and tail in Case (c) is just the movements of the information tail and front in Case (d). Moreover, it is noticed that Case (a) is a special case of Case (c), if no traffic flow moves in the opposite direction. Similarly, Case (b) is a special case of Case (d). Thus, this research takes Case (c) as a general case to study information spreading dynamics.

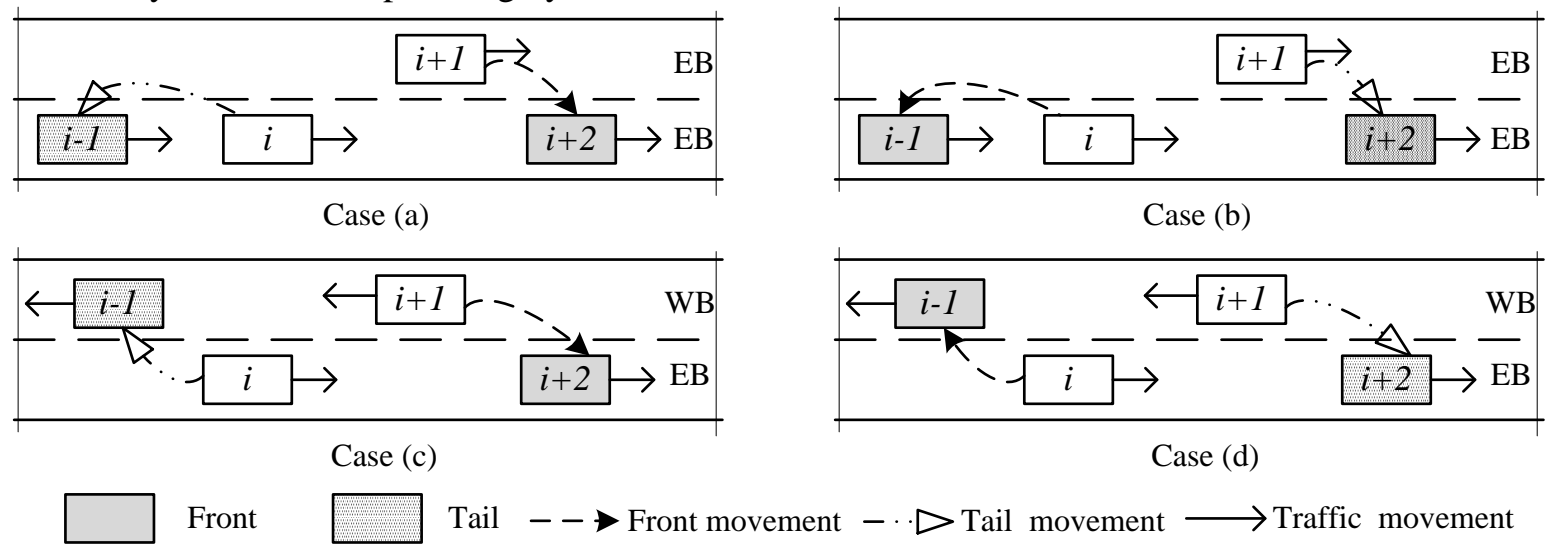

Figure 2. Four possible cases

\subsection{Intermittent transmission pattern}

To study the information spreading dynamics, we next explore the features of information spreading in temporal and spatial dimensions. A vehicle carrying the front or tail of an information wave is considered as a host vehicle. An information front/tail may move smoothly from a host vehicle to its neighbors through instantaneous or ferry transmission. The former indicates a series of uninterrupted wireless transmission when two vehicles are in the transmission range, such as the information transmission from vehicle $i$ to $i-1$ in Figure 1. The latter means that the wireless transmission is suspended, and an information front/tail will be carried by a host vehicle for a while until a new wireless connection is established, such as the information transmission from vehicle $i+2$ to $i+3$ in Figure 1. An information front/tail will experience a sequence of such instantaneous transmission followed by a ferry movement until it leaves a road segment. This study names such type of information propagation as intermittent transmission. Each combination of an instantaneous and a ferry movement is defined as an intermittent transmission pattern. Then, the process that an information front/tail goes through a road segment is consisted of a sequence of the intermittent transmissions. Clearly, the intermittent transmission provides a general view of information spreading over a temporal- spatial space. It happens more often than pure instantaneous or ferry transmissions in traffic flow. This study thus takes it as the base to build up our approaches.

The information propagation distance through instantaneous or ferry transmission is considered as random variables and denoted as $X$ and $Y$, respectively. Then, their expected values are denoted as $E(X)$ 
and $E(Y)$. Accordingly, the expected movement distance $(L)$ of an intermittent transmission pattern is measured by $L=(E(X)+E(Y))$, i.e., $L$ is equal to the summation of the expected instantaneous transmission distance and the expected ferry transmission distance. Sections 2.3 and 2.4 below present the approaches to measure $\mathrm{E}(\mathrm{X})$ and $\mathrm{E}(\mathrm{Y})$ under different traffic conditions according to (Du and Dao, 2015).

\subsection{Expected movement distance of instantaneous transmission $\mathbf{E}(\mathrm{X})$}

Du and Dao (2015) studied the expected distance of an instantaneous transmission in an intermittent transmission pattern on a road segment. Closed form formulations are developed to measure $E(X)$ under different traffic conditions. To make this study complete, we briefly talk about the ideas and the main results in this section. For more technical details, please refer the paper (Du and Dao, 2015). It is noticed that each hop of wireless transmission will succeed if the space $S$ between two consecutive vehicles is less than the transmission range $r$, i.e., $S \leq r$. Then the expected information movement distance of a single hop of wireless transmission is denoted as the expected value of $E(S \mid S \leq r)$, which can be calculated by Equation (1) given in (Du and Dao, 2015).

$$
E(S \mid S<r)=\int_{0}^{r} s f(s) d s / \int_{0}^{r} f(s) d s,
$$

where $f(s)$ is the probability density function of spacing headway/separation between two adjacent vehicles in a lane or across different lanes. Considering there are $\bar{k}$ hops of interrupted wireless transmission in an instantaneous transmission on the average, this study calculates the expected distance of instantaneous transmission $E(X)$ by Equation (2) below.

$$
E(X)=\bar{k} E(S \mid S<r)
$$

The expected number of hops $\bar{k}$ is calculated by Equation (3) and Equation (4) developed by (Du and Dao, 2015) under free traffic flow and congested flow, respectively.

$$
\begin{gathered}
\bar{k}=\sum_{\mathrm{k}=1}^{n} k \frac{(n-k) P^{k}(1-P)+P^{k}}{n+1}, \\
\bar{k} \leq \sum_{k=1}^{n} k \frac{(n-k)\left(2 P-P^{2}\right)(1-P)+2 P-P^{2}}{n+1}, \\
\bar{k} \geq \sum_{k=1}^{n} k \frac{(n-k)(k P-(k-1))(1-P)+k P-(k-1)}{n+1}, \\
P=p(S<r)=\int_{0}^{r} f(s) d s,
\end{gathered}
$$

where, $P$ represents the probability of a successful wireless communication between any two adjacent vehicles; $n$ is the number of possible hops in a vehicle platoon with $n+1$ vehicles. The idea used by (Du and Dao, 2015) to develop Equation (3) and Equation (4) is briefly presented as follows. The study labels the $n+1$ vehicles in a platoon from zero to $n$. The value of $\bar{k}$ is equal to the expectation of multiple probabilistic events, in each of which an instantaneous transmission starts from a vehicle at any place $\omega$ in a platoon, and then propagates a number of hops (any value $\leq \omega-1$ ) in a platoon. Du and Dao (2015) developed the formulations to calculate/approximate the probabilities of these events and obtained the formulations in Equation (3) and Equation (4). For more technical details, please refer the paper. Note that the proposed formulations are adaptive to the instantaneous transmission driven by either information front or tail in this study.

\subsection{Expected movement distance of ferry transmission $\mathrm{E}(\mathrm{Y})$}

$\mathrm{Du}$ and Dao (2015) also studied the expected distance of a ferry transmission in an intermittent transmission pattern on a road segment. This section briefly talks about the ideas and the main results, which will be applied to build up the approach in this study. We first consider a one-way road segment. As the space headway between two consecutive vehicles satisfy $S>r$, an information front or tail will be spread by a ferry transmission, which will be stopped as the space between two consecutive vehicles 
satisfies $S<r$. Therefore, the expected information propagation distance by a ferry transmission, $E(Y)$, can be calculated by Equation (6) given in (Du and Dao, 2015).

$$
E(Y)=v_{i} \times \frac{E(S \mid S>r)-r}{v_{i j}}=\frac{v_{i}}{v_{i j}}\left[\frac{\int_{r}^{+\infty} s f(s) d s}{1-\int_{0}^{r} f(s) d s}-r\right],
$$

where $E(S \mid S>r)$ represents the expected spacing headway/separation between two adjacent vehicles given a ferry transmission happens; $v_{i j}$ is the average relative speed between these two vehicles $i$ and $j$; $v_{i}$ is the speed of the vehicle $i$ carrying the information front or tail.

Next, we present the idea of (Du and Dao, 2015) to capture a ferry transmission as an information front or tail propagates on a two-way road segment, using an example shown in Figure 3. It is noticed that the ferry transmission on a two-way road segment can be a forward ferry or a backward ferry if the ferry starts at a vehicle moving along or opposite to the forward direction of information spreading. For example, Figure 3 (a) illustrates a forward ferry for an information front, in which a ferry transmission starts by vehicle $i$ moving along the forward direction of the information front, such as in EB; Figure 3 (b) illustrates a backward ferry for the information front, in which a ferry transmission starts by a vehicle $i$ moving opposite to the information front spreading direction, such as in WB.

Figure 3(a) indicates that a forward ferry by vehicle $i$ may have two possible scenarios: (i) stopping at vehicle $i+1$ moving in the same direction to the information propagation (ii) stopping at vehicle $i+2$ moving in the opposite direction to the information propagation. This study defines these two scenarios as $f_{1}$ and $f_{2}$, respectively. The expected distance $E\left(Y_{f 1}\right)$ or $E\left(Y_{f 2}\right)$ can be calculated by Equation (6), by applying $S$ or $S^{\prime}$ as $f_{1}$ or $f_{2}$ type of forward ferry transmission happens.

Figure 3(b) indicates that the backward ferry also involves two possible scenarios. (i) Vehicle $i$ carries the information back for a while later connects to vehicle $i+2$ before vehicle $i-1$ passes vehicle $i$; then the backward carrying distance is the movement of vehicle $i$ during this chasing process between vehicles $i$ and $i+2$. Hence, $E\left(Y_{f 3}\right)$ can be calculated by Equation (6). (ii) Vehicle $i$ meets vehicle $i-1$ before it connects to any other vehicles; then vehicle $i$ actually carries information back by the distance of $v_{i} E(S \mid S \leq r) / v_{i j}$; after vehicles $i$ and $i-1$ meet, the ferry transmission become forward ferry like the scenarios in Figure 3(a). Du and Dao (2015) ignored this scenario in measuring backward ferry, but considered it as the error involved in estimating $E(X)$ since the connection between vehicles $i$ and $i-1$ always exists during this period.

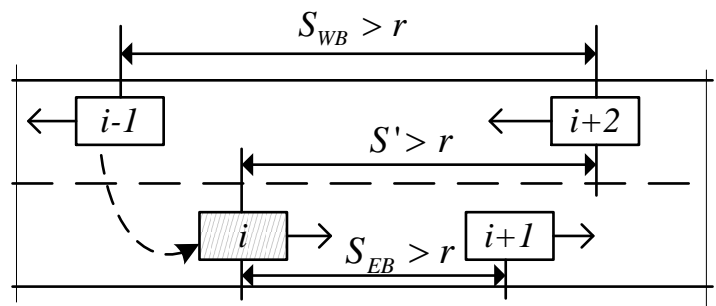

(a) Forward Ferry

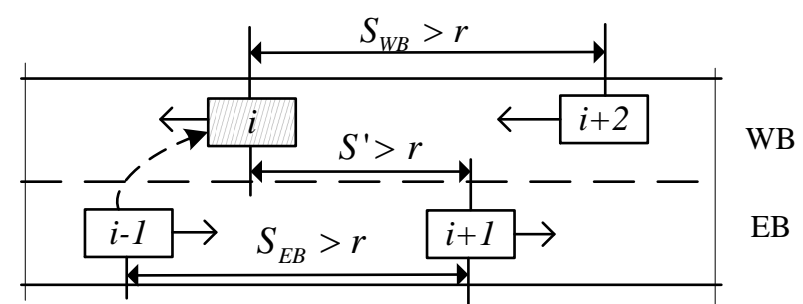

(b) Backward Ferry

$\longrightarrow$ Traffic flow moving direction

Figure 3 Ferry transmission of an information front by vehicle $i$ on a two-way road segment while vehicle

$i$ disconnects to vehicles $i+1$ and $i+2 . S^{\prime}$ : the space between two consecutive vehicles moving on different way. $S_{E B}$ and $S_{W B}$ : the space between two consecutive vehicles moving in EB and WB direction, respectively.

Integrating the discussions above, Equation (7) in (Du and Dao, 2015) presents the formulation to calculate the expected movement distance of a ferry transmission for information front.

$$
E(Y)=p_{1} * E\left(Y_{f 1}\right)+p_{2} * E\left(Y_{f 2}\right)-p_{3} * E\left(Y_{f 3}\right)
$$

where $E\left(Y_{f 1}\right), E\left(Y_{f 2}\right)$ and $E\left(Y_{f 3}\right)$ represents the expected distance of a forward and backward ferry transmission respectively, and $p_{1}, p_{2}$ and $p_{3}$ represent their possibilities to occur. Note that $E\left(Y_{f 1}\right)$, 
$E\left(Y_{f 2}\right)$ and $E\left(Y_{f 3}\right)$ can be calculated by Equation (6) with minor variations in the use of the space between two adjacent vehicles. $p_{1}, p_{2}$ and $p_{3}$ can be approximated by the probabilistic formulations developed in (Du and Dao, 2015) or by Monte Carlo simulations off-line.

Note: Equations (1) to (7), measuring $E(X)$ and $E(Y)$, rely on the function of $f(s)$, the probability density function of spacing headway between adjacent vehicles in a lane or across different lanes. This study provides detailed discussions regarding $f(s)$ in Section 3.2. In addition, the approaches and formulations presented above are also adaptive to a ferry transmission carrying information tail.

\subsection{Expected movement distance of an intermittent transmission pattern}

Table 1 below presents a summary of the formulations to measure movements of information front and tail. Mainly, the following points are highlighted. (i) The forward direction of information spreading in this study coincides with the forward direction of information front movement. Without loss of generality, we consider EB as the forward direction in all the examples of this study and assign positive (+) sign to a forward movement and negative (-) sign to a backward movement. (ii) Only forward (or backward) instantaneous transmission affects the location of the information front (or tail). (iii) The ferry transmission of information front involves three possible types: $f_{1}$, which is a forward ferry for an information front, starting from a vehicle moving in EB and ending on a vehicle moving in EB (i.e. $\mathrm{EB} \rightarrow \mathrm{EB}) ; f_{2}$, which is a type of forward ferry for information front, starting from a vehicle moving in EB and ending on a vehicle moving in $\mathrm{WB}$ (i.e. $\mathrm{EB} \rightarrow \mathrm{WB}$ ); $f_{3}$, which is a backward ferry for information front, starting from a vehicle moving in WB and ending at a vehicle moving in WB too (i.e. WB $\rightarrow \mathrm{WB}$ ). Similarly, there are three types of the ferry for an information tail. (iv) The expected movement distance of an intermittent transmission pattern for an information front or tail is the aggregation of an instantaneous and ferry movement of their own. (v) The proposed approaches do not differentiate lanes as information front or tail spreads on multiple lanes in same direction since $f(s)$ is the probability density function of the spacing headway/separation between adjacent vehicles in a lane or across different lanes.

Table 1. Information front and tail in-cell movement

\begin{tabular}{|c|c|c|}
\hline Movement & Information front & Information tail \\
\hline $\begin{array}{l}\text { Instantaneous } \\
\qquad E(X)\end{array}$ & $\begin{array}{c}\text { Forward: } \mathrm{EB} \Rightarrow+E\left(X_{f}\right)= \\
+\bar{k} E(S \mid S<r)\end{array}$ & $\begin{array}{c}\text { Backward: WB } \Rightarrow-E\left(X_{t}\right)= \\
\quad-\bar{k} E(S \mid S<r)\end{array}$ \\
\hline \multirow{2}{*}{$\begin{array}{l}\text { Ferry } \\
E(Y)\end{array}$} & $\begin{array}{c}f_{1}:(\mathrm{EB}) \rightarrow(\mathrm{EB}) \Rightarrow+E\left(Y_{f_{1}}\right) \\
f_{2}:(\mathrm{EB}) \rightarrow(\mathrm{WB}) \Rightarrow+E\left(Y_{f_{2}}\right) \\
f_{3}:(\mathrm{WB}) \rightarrow(\mathrm{WB}) \Rightarrow-E\left(Y_{f_{3}}\right)\end{array}$ & $\begin{aligned} f_{1}:(\mathrm{WB}) & \rightarrow(\mathrm{WB}) \Rightarrow-E\left(Y_{f_{1}}\right) \\
f_{2}:(\mathrm{WB}) & \rightarrow(\mathrm{EB}) \Rightarrow-E\left(Y_{f_{2}}\right) \\
f_{3}:(\mathrm{EB}) & \rightarrow(\mathrm{EB}) \Rightarrow+E\left(Y_{3}\right)\end{aligned}$ \\
\hline & $\begin{array}{c}E\left(Y_{f}\right)=p_{1} E\left(Y_{f 1}\right)+p_{2} E\left(Y_{f 2}\right) \\
-p_{3} E\left(Y_{f 3}\right)\end{array}$ & $\begin{array}{c}E\left(Y_{t}\right)=-p_{1} E\left(Y_{f 1}\right)-p_{2} E\left(Y_{f 2}\right) \\
+p_{3} E\left(Y_{f 3}\right)\end{array}$ \\
\hline Pattern & $L_{f}=\left|E\left(X_{f}\right)+E\left(Y_{f}\right)\right|$ & $L_{t}=\left|-E\left(X_{t}\right)+E\left(Y_{t}\right)\right|$ \\
\hline \multicolumn{3}{|c|}{$E(X)$ and $E(Y)$ are given in Equations (2) and (7) respectively; $L_{t}$ and $L_{f}$ are expected value } \\
\hline
\end{tabular}

\section{Methodology for Information Spreading Dynamics}

This section presents our methodology to capture information spreading dynamics as it propagates via VANET on a road segment. Recall information spreading dynamics is represented by the movements of information front and tail or the variation of information coverage over a temporal- spatial space. Initiated by the cell transmission model (CTM) developed by (Daganzo, 1994), this study seeks to establish an information-traffic coupled cell transmission model (IT-CTM) which can discretely describe information propagation dynamics on top of traffic flow dynamics in a temporal-spatial space. More exactly, this 
study develops methods to track the locations of information front and tail in the cells of IT-CTM in discrete time steps, and then measures the corresponding information coverage. Thus, the IT-CTM is built upon several key elements including determining the cell length, tracking inner-cell and inter-cell information propagation as well as information merging. In what follows, we present our methods in technical details.

\subsection{Information-traffic coupled cell}

To develop IT-CTM approach, we first briefly review CTM developed in (Daganzo, 1994), which has been well accepted as a discrete model to capture macroscopic traffic flow dynamics. Basic CTM splits a road segment into a number of cells. Each cell is with a length $\Delta x$; the number of cells is determined by $J=L / \Delta x$. According to the CTM theory, the number of vehicles in cell $j$ at time $t_{i}$ is calculated in the equation below:

$$
n_{j}\left(t_{i}\right)=\mathrm{n}\left(t_{i-1}\right)+y_{j}\left(t_{i-1}\right)-y_{j+1}\left(t_{i-1}\right),
$$

where $n_{j}\left(t_{i}\right)$ is the number of vehicles in cell $j$ at $t_{i} ; n_{j}\left(t_{i-1}\right)$ is the number of vehicles in cell $j$ at $t_{i-1}$, $y_{j}\left(t_{i-1}\right)$ is the inflow from cell $\mathrm{j}-1$ to cell $j$ at $t_{i-1}$, and $y_{j+1}\left(t_{i-1}\right)$ is the outflow from cell $j$ to cell $j+1$ at $t_{i-1}$. Moreover, the inflow $y_{j}\left(t_{i-1}\right)$ and the outflow $y_{j+1}\left(t_{i-1}\right)$ are calculated by Equations (9) and (10) respectively.

$$
\begin{gathered}
y_{j}\left(t_{i-1}\right)=\min \left[n_{j-1}\left(t_{i-1}\right), q_{j}^{m} \Delta t, \omega_{b} / v_{f}\left(k_{j a m} \Delta x-n_{j}\left(t_{i-1}\right)\right)\right], \\
y_{j+1}\left(t_{i-1}\right)=\min \left[n_{j}\left(t_{i-1}\right), q_{j+1}^{m} \Delta t, \omega_{b} / v_{f}\left(k_{j a m} \Delta x-n_{j+1}\left(t_{i-1}\right)\right)\right],
\end{gathered}
$$

where $q_{(.)}^{m}$ is the maximum capacity of traffic flow at cell (.); $\omega_{b}$ is the backward shock wave speed; $v_{f}$ is the free flow traffic speed; $k_{j a m}$ is the jam density.

This study next develops the IT-CTM to capture information spreading dynamics. The basic idea is shown in Figure 4. Specifically, besides traffic volume and its evolvement over time, we hope the cell of IT-CTM can also discretely track the spreading of information front and tail in a temporal-spatial space. Accordingly, this study introduces two indicators $f_{j}(t)$ and $\tau_{j}(t)$ below to indicate whether information front or tail moves in cell $j$ at $t^{t h}$ time interval. Section 3.4. provides technical details to identify them.

$$
\begin{aligned}
& f_{j}(t)=\left\{\begin{array}{lc}
1, & \text { information front stops at cell } j \text { by the end of time } t \\
0, & \text { otherwise }
\end{array}\right. \\
& \tau_{j}(t)=\left\{\begin{array}{lc}
1, & \text { information tail stops at cell } j \text { by the end of time } t \\
0, & \text { otherwise }
\end{array}\right. \\
& \begin{array}{lll}
\text { Cell } j-1 & \text { Cell } j & \text { Cell } j+1
\end{array} \\
& t _ { i - 1 } \longdiv { [ n _ { j - 1 } ( t _ { i - 1 } ) , f _ { j - 1 } ( t _ { i - 1 } ) , \tau _ { j - 1 } ( t _ { i - 1 } ) ] \sqrt [ y _ { i } ( t _ { i - 1 } ) ] { } \rangle [ n _ { j } ( t _ { i - 1 } ) , f _ { j } ( t _ { i - 1 } ) , \tau _ { j } ( t _ { i - 1 } ) ] [ y _ { i + 1 } ( t _ { i - 1 } ) \rangle [ n _ { j + 1 } ( t _ { i - 1 } ) , f _ { j + 1 } ( t _ { i - 1 } ) , \tau _ { j + 1 } ( t _ { i - 1 } ) ] } \\
& \begin{array}{l|l|l|l|}
\cline { 2 - 3 } t_{i} & {\left[n_{j-1}\left(t_{i}\right), f_{j-1}\left(t_{i}\right), \tau_{j-1}\left(t_{i}\right)\right]} & {\left[n_{j}\left(t_{i}\right), f_{j}\left(t_{i}\right), \tau_{j}\left(t_{i}\right)\right]} & {\left[n_{j+1}\left(t_{i}\right), f_{j+1}\left(t_{i}\right), \tau_{j+1}\left(t_{i}\right)\right]} \\
\hline
\end{array}
\end{aligned}
$$

Figure 4. IT-CTM

To achieve this goal, we will revisit the selection of the cell length, and formulate information spreading over cells beyond the basic CTM model. The first critical issue is how to select the cell length for IT-CTM so that it is adaptive to both information and traffic flow dynamics. It is known that the cell length in CTM is usually determined by the product of free flow traffic speed $\left(v_{f}\right)$ and pre-defined sample time interval $(\Delta t)$, i.e., $v_{f} \times \Delta t$. Namely, there is few vehicles moving through a cell during $\Delta t$ so that traffic state in each cell during $\Delta t$ can be considered as static. However, this cell length may be too short to cover an intermittent transmission pattern, and then prevents using our methods to capture information propagation via VANET. Recall this study considers that the movement of the information 
tail/front is consisted of a sequence of intermittent transmissions, each including an instantaneous transmission and a ferry in Section 2.2. Thus, to capture information spreading, a cell of IT-CTM is required to cover at least one intermittent transmission pattern for information front or tail during a sample time step. Accordingly, the cell length of the IT-CTM is then given by $\Delta x=\max \left\{a L_{f}, a L_{t}, v_{f} \Delta t\right\}$, where $a \geq 1$ is a constant integer value. As $a=1$, a cell of IT-CTM covers at least one intermittent transmission pattern of information front or tail. As $a>1$, at least $a$ number of intermittent transmission patterns of information front or tail will be covered in a IT-CTM cell. Since this proposed study assumes homogeneous traffic flow for inner-cell transmission (see Section 3.2), a bigger value of $a$ can be selected as traffic flow on a road segment is more close to homogeneous, otherwise a smaller value of $a$ is preferred. Considering that $\Delta t$ in CTM is a flexible value, we can always find a value of $\Delta t$ so that $\Delta t v_{f}=\Delta x=\max \left\{a L_{f}, a L_{t}\right\}$. As a result, to determine $\Delta x$ becomes to determine $\Delta t$ given the values of $a L_{f}, a L_{t}, v_{f}$. The above idea is presented by Equation (13) below.

$$
\Delta x=\max \left\{a L_{f}, a L_{t}, v_{f} \Delta t\right\} \Leftrightarrow \Delta t=\max \left\{a L_{f}, a L_{t}\right\} / v_{f}
$$

Moreover, the proposed approach can be extended to a one-way or two-way road segment with multiple lanes. We show this advantage through different scenarios below. For a two-way road segment with one lane in each way, we adopt the IT-CTM for each traffic direction separately, and make cells on different ways but aligned with the same length. Considering traffic flow on different traffic way might be different which results in the cell lengths $\Delta x^{\prime}$ and $\Delta x^{\prime \prime}$ respectively, we choose the cell length, $\Delta x=$ $\max \left\{\Delta x^{\prime}, \Delta x^{\prime \prime}\right\}$ for both directions on a two-way road segment.

For a one-way road segment with $I$ number of lanes, traffic flow in each lane can be tracked by a CTM model by making CTM cells on different lanes aligned with the same cell length, i.e., $\Delta x=\max \left\{\Delta x_{i}, i=\right.$ $1, \ldots, I\}$. The summation of the traffic counts in aligned cells across all lanes give us a traffic count in a cell of IT-CTM. The extended CTM model developed by (Carey et al., 2014), which allows multiple lane and lane-changing, may be used here to track traffic flow more accurately. Accordingly, information flow spreading can still be captured by the IT-CTM since this study do not differentiate lanes in tracking traffic information spreading (refer to point (v) in section 2.5).

Clearly, combining the approaches for the above two scenarios, we can extend this study to a two-way road segment with multiple lanes in each way. Note that although the proposed IT-CTM does not require a uniform length among all cells in longitudinal direction, uniform cell length will facilitate the discussion and calculation, thus it is adopted in this study. To complete the approach of the IT-CTM, next sections formulate the information propagation in a cell and between cells.

\subsection{Inner-cell information movement state}

This study next presents our approach to identify the information movement in a cell relying on the ITCTM model, considering homogeneous traffic flow in a cell. The analyses in Section 2.2 and 2.3 recognized that instantaneous and ferry transmissions may bring information front and tail back and forth over the time. Then each intermittent transmission pattern may present three possible states: forward, backward or stationary given predefined information spreading direction. Accordingly, this study examines the movement direction (sign) of an intermittent transmission pattern.

Specifically, for the information front, the instantaneous transmission is always positive because only forward instantaneous transmission affects its spreading. However, ferry transmission may be positive, negative or zero under different traffic conditions. On the other hand, for the information tail, instantaneous transmission is always negative since only backward instantaneous transmission is effective, and ferry transmission may also be negative, positive or zero under different traffic conditions. That is to say, the movement of an intermittent transmission pattern, $L_{f}=E\left(X_{f}\right)+E\left(Y_{f}\right)\left(\right.$ or $\left.L_{t}=-E\left(X_{t}\right)+E\left(Y_{t}\right)\right)$ may be negative, positive or zero, each of which indicates a scenario below to describe the inner-cell movement pattern for an information front or tail. 
Forward Cell: If $L_{f}>0$ or $L_{t}>0$ for information front or tail, then information front or tail moves forward. Therefore, after an intermittent propagation pattern, information front (or tail) moves forward by a distance $L_{f}\left(\right.$ or $\left.L_{t}\right)$.

Backward Cell: If $L_{f}<0$ or $L_{t}<0$ for information front or tail, then information front or tail moves backward. Therefore, after an intermittent propagation pattern, information front (or tail) moves back by a distance $L_{f}$ (or $\left.L_{t}\right)$.

Stationary Cell: If $L_{f}=0$ or $L_{t}=0$ for information front or tail, then information front or tail will stay stationary.

Note that the IT-CTM considers homogeneous traffic flow in a cell. Thus, once a cell is identified in forward or backward state, the forward or backward intermittent propagation pattern will repeat in the cell until it reaches to the boundary of the cell. However, if a cell is identified in stationary state, then information front or tail will stay at the same point in this cell during $\Delta t$. Accordingly, this study estimates the inner-cell delay, $E\left(T_{j}\right)$, for an information front or tail moving in a forward or backward cell by Equation (14) below.

$$
E\left(T_{j}\right)=\pi \frac{\Delta x}{\left|L_{*}\right|}
$$

where $\pi$ represents the average delay of an intermittent transmission pattern, and $\frac{\Delta x}{\left|L_{*}\right|}$ provides the average number of intermittent transmissions experienced by an information front $\left(L_{*}=L_{f}\right)$ or tail $\left(L_{*}=L_{t}\right)$ to go through the cell.

Given the intermittent transmission pattern discussed in Table 1, and the formulations given in Equations (1)-(7), which calculate the movement distance of a ferry and instantaneous transmissions, this study develops Equations (15) and (16) below to respectively calculate inner-cell delay $E\left(T_{j}\right)$ for an information front and tail to going through cell $j$ in case (c), shown in Figure 2.

$$
\begin{array}{lr}
E\left(T_{j}\right)=\left[\bar{k} * \theta+p_{1} \frac{E\left(Y_{f 1}\right)}{v}+p_{2} \frac{E\left(Y_{f 2}\right)}{v}+p_{3} \frac{E\left(Y_{f 3}\right)}{v}\right] & \text { For Information } \\
\times\left[\frac{\Delta x}{\left|E(X)+p_{1} * E\left(Y_{f 1}\right)+p_{2} * E\left(Y_{f 2}\right)-p_{3} * E\left(Y_{f 3}\right)\right|}\right], & \text { Front } \\
E\left(T_{j}\right)=\left[\bar{k} * \theta+p_{1} \frac{E\left(Y_{f 1}\right)}{v}+p_{2} \frac{E\left(Y_{f 2}\right)}{v}+p_{3} \frac{E\left(Y_{f 3}\right)}{v}\right] & \text { For Information } \\
\times\left[\frac{\Delta x}{\left|-E(X)-p_{1} * E\left(Y_{f 1}\right)-p_{2} * E\left(Y_{f 2}\right)+p_{3} * E\left(Y_{f 3}\right)\right|}\right], & \text { Tail }
\end{array}
$$

where $\theta$ is the delay resulting from one hop of wireless transmission; $v$ represents the traffic speed of the carrying vehicle, which can be substituted by the average speed of vehicles moving in the same direction on the road. Note that the first bracket in each equation provides the value of $\pi$, which is the summation of the expected delay of instantaneous transmission, $\bar{k} * \theta$ and the expected delay of a ferry transmission involving three different types of ferry transmissions discussed in Table 1 . The second bracket in each equation provides the value of $\frac{\Delta x}{\left|L_{*}\right|}$.

Specifically, the delay of an intermittent transmission results from both instantaneous transmission and ferry transmission. If we consider the average delay of each hope of wireless transmission as a constant value $\theta$ under given wireless communication standards such as IEEE 802.11p for DSRC (Jiang et. al., 2006; Al-Sultan et. al., 2014), then the delay resulting from instantaneous transmission in an intermittent transmission is equal to the product of the average number of hops $\bar{k}$ and the delay at each hop, i.e., $\bar{k} * \theta$. Next, the delay resulting from a ferry transmission for a front or tail is related to three possible ferry transmissions $\left(f_{1}, f_{2}\right.$, and $\left.f_{3}\right)$ discussed in Table 1 . Each type of ferry occurs with certain probability. Then, the average delay resulting from ferry transmission is equal to their expectation, i.e., $p_{1} \frac{E\left(Y_{f 1}\right)}{v}+$ 
$p_{2} \frac{E\left(Y_{f 2}\right)}{v}+p_{3} \frac{E\left(Y_{f 3}\right)}{v}$. Recall that the formulations to capture $E\left(Y_{f 1}\right), E\left(Y_{f 2}\right), E\left(Y_{f 3}\right), \bar{k}$, as well as $p_{1}, p_{2}$ and $p_{3}$ have been discussed in Equations (1)-(7).

Moreover, if an information front or tail is in a forward or backward cell but it cannot go through a cell during a sample time interval $\Delta t$, then we need to determine where the information front or tail will stop in a cell at the end of the time interval. We denote the in-cell location of an information front or tail as $d_{j}$. Considering information propagates by a uniform speed in a cell, Equation (17) presents our approach to measure $d_{j}$.

$$
d_{j}(\mathrm{t})=\left\{\begin{array}{cc}
\frac{\Delta x}{E\left(T_{j}\right)} * \Delta t & \text { if cell } \mathrm{j} \text { is a forward cell } \\
\Delta x-\frac{\Delta x}{E\left(T_{j}\right)} * \Delta t & \text { if cell } \mathrm{j} \text { is a backward cell }
\end{array}\right.
$$

Note: to identify the state of the intermittent information propagation in a cell, we calculate $E(X)$ and $E(Y)$ by Equations (1) to (7). But these equations require a known function of $f(s)$ (recall that it represents the probability distribution of the spacing between adjacent vehicles in a lane or across different lanes). This study identifies the function of $f(s)$ by the macroscopic traffic flow information provided by IT-CTM and LOS defined in HCM 2010(2010). Specifically, IT-CTM is able to track traffic volume $n_{j}(t)$ in each cell at each sample time interval by Equation (8). Thus, with a given cell length, we can estimate traffic density in each cell by $\bar{\rho}_{j}\left(t_{i}\right)=n_{j}(t) / \Delta x$. According to the LOS criteria given in (HCM 2010, 2010), we are able to estimate if the traffic flow in a cell is under free flow or congested flow. Next, according to existing study on the spacing headway distribution (Zhang et. al., 2007; Wang, 2012), this study considers $f(s)$ in the cell follows exponential distribution with mean and variance equal to $1 / \bar{\rho}_{j}(t)$, if a free flow presents in a cell (such as traffic density is in the range of 0 to $45 \mathrm{pc} / \mathrm{mi} / \mathrm{l}$ ); or $f(s$ ) in the cell follows log-normal distribution with mean equal to $1 / \bar{\rho}_{j}(t)$ and the variance obtained from historical data, if a congested flow presents (such as traffic density is range of 45 to $190 \mathrm{pc} / \mathrm{mi} / \mathrm{l}$ ) in a cell. In this way, this study bridges the macroscopic traffic flow information with information spreading without knowing accurate microscopic vehicle distribution, so that Equations (1) - (7) can be used to calculate $E(X)$ and $E(Y)$ in the cell.

\subsection{Inter-cell information spreading}

This research further studies the information spreading between cells under the approach of the ITCTM, i.e., inter-cell information spreading in a chain of information cells. Here, we consider nonhomogeneous traffic flow among cells. Figure 5 illustrates that the possible combinations of the states between two adjacent cells, each of which might in a forward, backward or stationary state. Accordingly, the information front (or tail) spreading from one cell to its neighbor cells will be determined by their states. Theoretically, there are $3^{2}=9$ cases. Using the example given in Figure 5 , this study develops the regulations for inter-cell information spreading, i.e. the propagation from cell $j$ to $j+1$ or $j-1$.

Scenario I. Cell $j$ is a stationary cell and cell $j+1$ is either a forward, backward, or stationary cell. Since information won't propagate in a stationary cell, we consider information front or tail will stay inside of cell $j$ during the corresponding time interval $\Delta t$.

Scenario II. Cell $j$ is a forward cell, and cell $j+1$ is in any of the three possible states. For Scenario II (a) in Figure 5, the successive cell is a forward cell, and then information front or tail will enter cell $\mathrm{j}+1$ and propagate forward. For Scenario II (b) and (c) in Figure 5, where the successive cell is a backward or stationary cell, this study considers that the information front/tail will stay at the boundary between cell $j$ and $j+1$ at the end of the time interval $\Delta t$ since it won't be able to move forward.

Scenario III. cell $j$ is a backward cell and cell $j-1$ instead of cell $j+1$ will affect the information propagation of a front or a tail in a time interval. For Scenario III (a) in Figure 5, the successive cell is a 
backward cell, and then information front or tail will enter cell $j-1$ and propagate backward. For Scenario III (b) in Figure 5, we consider the information tail or front will stay at the boundary of cell $j-$ 1 and $j$ at the end of a time interval with the same reason as scenarios Scenario II (b) and (c).

(a)

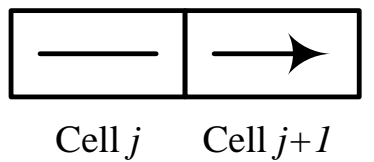

(b)

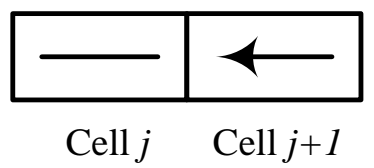

(c)

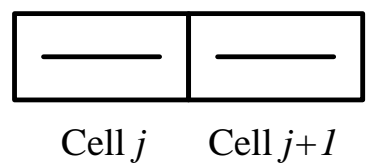

(a)

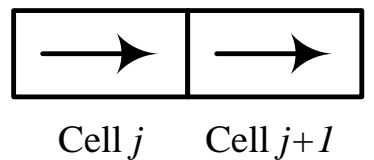

(b)

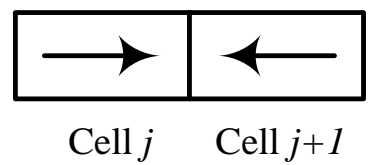

(c)

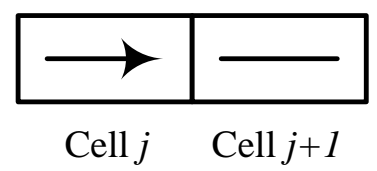

Scenario II: Cell $j$ is a forward cell and $j+1$ are in different states

(a)

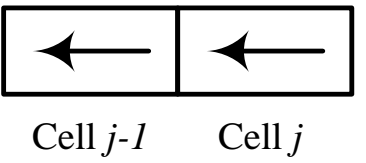

(b)

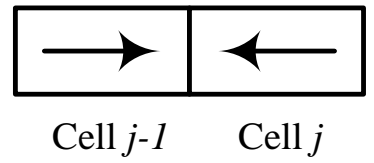

(c)

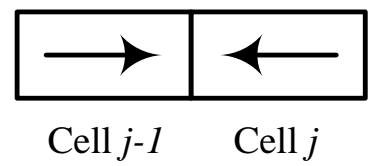

Scenario III: Cell $j$ is a backward cell and $j-1$ are in different states

Figure 5. Inter-cell movement

The above discussions indicate that it is possible that the information front or tail stops at the boundary of two cells rather than inside a cell by the end of a time interval, such as Scenario II (b) and (c), and Scenario III (b) and (c) in Figure 5. Actually, Scenario II (a) and Scenario III (a) in Figure 5 are also the possible cases that the information front or tail just stops at the boundary of two cells after it experiences several in-cell movements during time interval $\Delta t$.

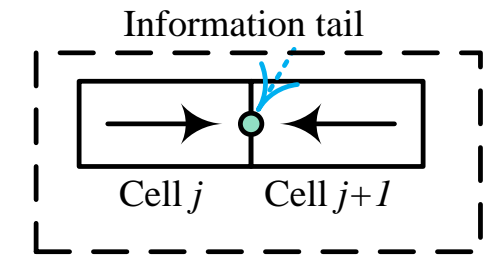

At the end of the $(\mathrm{t}-1)^{\text {th }}$ time step

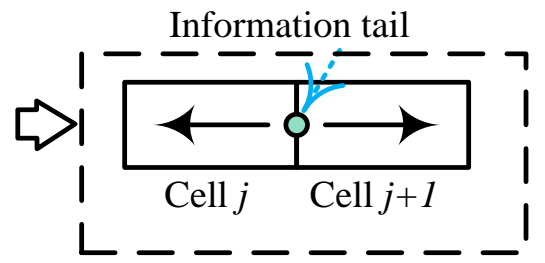

At the start of the $(\mathrm{t})^{\text {th }}$ time step

Figure 6. Illustration of cell merging

We define the situation as a boundary case if at the beginning of a time interval, an information front (or tail) sits at the boundary of two adjacent cells under different states. It may cause a dilemma to decide which direction/state the information front or tail should follow. An example is shown in Figure 6, in which an information front/tail stops at the boundary of cells $j$ and $j+1$ at the end of the $(t-1)^{t h}$ time interval. Then, at the $t^{t h}$ interval, cell $i$ changes to a backward cell and cell $j+1$ changes to a forward cell, previous regulations for inter-cell movement meet the difficulty to decide which direction the information front/tail should go further during the $t^{\text {th }}$ time interval. To address this issue, this study proposes to unite the corresponding two cells as one cell so that information front/tail can be treated as staying inside a unit cell. Then, by following inner-cell information propagation method provided in Section 3.3, we identify that the state of the intermittent transmission pattern in the united cell. Namely, if the united cell is a forward cell, the information front/tail will enter cell $j+1$ and propagate forward. If the united cell is a backward cell, the information front/tail enter cell $j$ and propagate backward. If the united cell is a stationary cell, the information front/tail will stay at the boundary of cells $j$ and $j+1$ during the corresponding time interval $\Delta t$. 


\subsection{Information spreading dynamics along cells}

Several forward cells between cell 1 to cell $j$

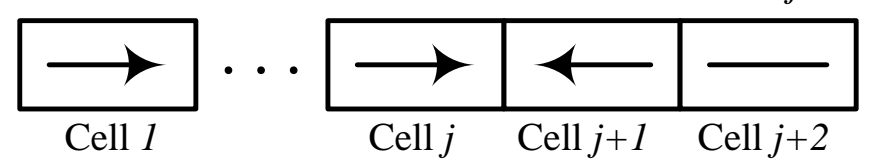

Figure 7. A chain of information cells on a road segment

This section studies information spreading along a chain of cells (see Figure 7) according to the innercell and inter-cell movement. More exactly, we seek to identify the location of information front and tail at the end of each sample time interval (i.e. the values of $f_{j}\left(t_{i}\right)$ and $\tau_{j}\left(t_{i}\right)$ ). Considering information front and tail propagate similarly, we take information front as an example to present our approach. Assuming that the information front is located inside cell $\mathrm{j}^{5}$, at the beginning at time $\mathrm{t}_{0}$, it will propagate by the following procedure.

Step 0: $t=t_{0}$ determine the states of cells the approach given in Section 3.2

Step 1: Check if belongs to the boundary case

1.1 No. The front locates at $d_{j}\left(t_{0}-\Delta t\right)$ in cell $j$ at the beginning of $t_{0}$. Go to Step 2 .

1.2 Yes. The front locates at the boundary of cell $j-1$ and cell $j\left(\right.$ i.e. $d_{j}\left(t_{0}-\Delta t\right)=0$ ), unite cells $j-1$ and $j$.

- If the united cell is a forward cell. Information front enters cell $j . j=j$, and go to step 2 .

- If the united cell is a backward cell. Information front enters cell $j-1 . j=j-1$, and go to step 2.

- If the united cell is a stationary cell. Information front stays at the boundary. $f_{j-1}(t)=1, f_{j}(t)=0, d_{j}(t)=0$, and go to step 4 .

Step 2: Determine inner-cell movement

2.1 If cell $j$ is stationary, then $f_{j}(t)=1$, information front stops inside cell $j$, and go to Step 4 .

2.2 If cell $j$ is forward, then information front propagates forward. We determine,

- If inner-cell delay $E\left(T_{j}\right)<\Delta t, f_{j}(t)=0$, information front goes through cell $j$ and go to Step 3.1

- If inner-cell delay $E\left(T_{j}\right)>\Delta t, f_{j}(t)=1$, information front stops at $d_{j}(t)$ in cell $j$, and go to Step 4

- If inner-cell delay $E\left(T_{j}\right)=\Delta t, f_{j}(t)=1$, information front stops at the boundary of cell $j$ and cell $j+1, d_{j+1}(t)=0$, and go to Step 4 .

2.3 If cell $j$ is backward, then information front propagates backward. We determine

- If inner-cell delay $E\left(T_{j}\right)<\Delta t, f_{j}(t)=0$, information front goes through cell $j$ and go to Step 3.2

- If inner-cell delay $E\left(T_{j}\right)>\Delta t, f_{j}(t)=1$, information front stops at $d_{j}(t)$ in cell $j$, and go to Step 4

- If inner-cell delay $E\left(T_{j}\right)=\Delta t, f_{j}(t)=0, f_{j-1}(t)=1$, information front stay at the boundary of cell $j-1$ and cell $j, d_{j}(t)=0$, and go to Step 4 .

Step 3: Determine inter-cell movement

3.1 If cell $j+1$ is forward cell, $\Delta t=\Delta t-E\left(T_{j}\right), j=j+1$, go to Step 2.2.

If cell $j+1$ is stationary or backward $f_{j}(t)=1, d_{j+1}(t)=0, j=j+1$. Information front

\footnotetext{
${ }^{5}$ We assume the front has not fully gone through cell $\mathrm{j}$.
} 
stops at the boundary of cell $j$ and cell $j+1$, and go to Step 4 .

3.2 If cell $j-1$ is backward cell, then $\Delta t=\Delta t-E\left(T_{j}\right), j=j-1$, go to Step 2.3.

If cell $j-1$ is stationary or forward, then $f_{j-1}(t)=1, d_{j}(t)=0, j=j-1$; information front stops at the boundary of cell $j-1$ and cell $j$ so go to Step 4 .

Step 4: $t_{0}=t_{0}+\Delta t$, go to Step 0 .

Note: (i) At the beginning of each time interval, if an information front/tail stays inside a cell, its inner-cell delay is scaled as $\frac{\Delta x-d_{j}\left(t_{0}-\Delta t\right)}{\Delta x} E\left(T_{j}\right)$ (or $\frac{d_{j}\left(t_{0}-\Delta t\right)}{\Delta x} E\left(T_{j}\right)$ ) for forward (or backward) cell $j$. (ii) At the end of each time interval, if an information front/tail stops inside a cell, we set $f_{j}(t)=1$; if an information front/tail stops at the boundary of cell $j-1$ and cell $j$, we set $f_{j-1}(t)=1, f_{j}(t)=0$ and $d_{j}(t)=0$. (iii) The procedure above can be used to track the spreading dynamics of information tail too.

The proposed procedure above dynamically updates the states of $f_{j}(t)$ and $\tau_{j}(t)$ for all cells in the ITCTM built upon a road segment. As a result, the IT-CTM model produces a time series vector as below to demonstrate the information spreading dynamics on the road segment.

$$
\left\{\left(n_{1}(t), f_{1}(t), \tau_{1}(t)\right), \ldots,\left(n_{J}(t), f_{J}(t), \tau_{J}(t)\right)\right\}, t=1,2,3 \ldots
$$

According to the time series vector, this study can also capture the dynamic coverage of the information spreading on the road; i.e., the percentage of cells (or vehicles) which are covered by the information on a road. More exactly, if cell $i$ holds the information tail and cell $j$ holds the information front, then all the cells (or vehicles) between cell $i$ and $j$ are covered by the information. To perform mathematical calculation, this study introduces another indicator defined by Equation (19).

$$
c_{\omega}(t)=\left\{\begin{array}{cc}
1, & \text { if } f_{j}(t)=1 \text { and } \tau_{i}(t)=1, i \leq \omega \leq j \\
0, & \text { otherwise }
\end{array}\right.
$$

Clearly, $c_{\omega}(t)=1$ indicates the cell as well as the vehicles in the cell is (are) covered by the information. Then the information coverage $c(t)$ over a segment at time stamp t is can be calculated by Equation (20).

$$
c(t)=\frac{\sum_{\omega=1}^{J} c_{\omega}(t)}{J}, \text { or } c(t)=\frac{\sum_{\omega=1}^{J} n_{\omega}(t) c_{\omega}(t)}{\sum_{\omega=1}^{J} n_{\omega}(t)} t=1,2,3 \ldots
$$

$c(t)$ evolves over time with the maximum value equal to 1 , which indicates all cells (or all vehicles) on the segment are covered, and the minimum value is 0 , which means the information has not arrived at this road segment or the information already leaves this segment. Therefore, the proposed IT-CTM is able to track the information coverage dynamics over a road segment, and provide us knowledge about how many vehicles on a road segment are informed by a traffic event after it launches on a road. This is valuable for the applications of VANET such as traffic event/hazardous warning.

\subsection{Information merging}

In reality, multiple pieces of information with similar or same messages may arrive at a road segment simultaneously or sequentially. As they come simultaneously, the IT-CTM treats them as one piece of information since they together form a single information front and tail. As they arrive sequentially, the propagation of two pieces of information may form two separate information waves with different information fronts and tails. More exactly, the later one may propagate either faster or slower than the one arriving earlier. Consequently, these two pieces of information may either meet at certain location and time, or always propagate separately. The study proposes two more regulations so that IT-CTM approach is able to address both situations.

First, if the front of the information coming later is behind the tail of the information coming earlier, this study tracks the information fronts and tails for both pieces of information at each $\Delta t$. The information coverage at each time interval is the summation of the coverage of the two pieces of information. Second, if the front of the information coming later catches up the tail of the information coming earlier, the wave of the two pieces of information merge to one. Namely, this study takes the front 
of the information coming earlier as the new front and the tail of information coming later as the new tail of the information wave on the road segment; accordingly, the tail (or front) of the information coming earlier (or later) vanishes. Our numerical experiments below demonstrate the capability of the IT-CTM to track the spreading of multiple pieces of information sequentially arriving at a road segment.

\section{Experiment and analysis}

The applicability and accuracy of the IT-CTM to capture information spreading dynamics are demonstrated through the numerical experiments built upon both field data and simulation data. The following section presents our data set, testbed, experiment design and main results.

\subsection{Input data and testbeds}

The numerical experiments are conducted on two testbeds to cover one-way and two-way road segments with fluctuated entering traffic flow. More exactly, the experiments on one-way road segments used vehicle trajectory data obtained from both NGSIM and Paramics simulation. The NGSIM trajectory data was collected during rush hour between 8:20 a.m. to 8:35 a.m. on June 15, 2005, for a 2100 feet segment with 5 main lanes on U.S. Highway 101 (Hollywood Freeway) in Los Angeles, California. Vehicles travel through the segment from North to South. The average traffic flow is $7604 \mathrm{vph}$. Since the road length of NGSIM data is too short to efficiently demonstrate the information spreading dynamics, this study built a road segment model in Paramics and used the simulated traffic flow data to validate the performance of the IT-CTM approach. The road segment built in Paramics is three miles long and with two lanes. The flow capacity per lane is $2400 \mathrm{vph}$. The entering flow of the road segment is randomly generated through the RNG (random number generator) in Paramics with the predefined mean $(\mu)$ and derivation $(\sigma)$ which are presented in Table 2. All the simulation experiments in Paramics were run for 40 minutes and the first 15 minutes are treated as the warm-up time. Both NGSIM and simulation data include essential attributes including vehicle ID, time step, local Y (distance from the beginning of road segment), vehicle velocity, lane number, space headway and time headway.

\subsection{Experiment design}

To demonstrate the accuracy of the IT-CTM approach, all the experiments will track the locations of information front and tail in each time interval through the IT-CTM and the simulation trace. Accordingly, $c(t)^{M}$ and $c(t)^{S}$ are introduced to represent the information coverage obtained from the ITCTM and the ground truth observed from the simulation at each time interval. Below explains the details of the experimental procedures.

Our experiments first tracked the movement of information front and tail according to vehicle trajectory data obtained from NGSIM filed collection or simulation in Paramics. Once a piece of information arrives to the start of the road segment, the information spreads smoothly as two consecutive vehicles are well connected (i.e., the space between two vehicles are less than or equal to the transmission range $r$ ) until a disconnection happens. Accordingly, initial information front and tail are generated. Next, the corresponding information coverage $c(t)^{S}$ may be tracked and updated in each time interval $\Delta t$ according to the dynamic movement of the information front and tail. Here, $r$ is a parameter determined by the method in (Du and Dao, 2015) considering the average traffic condition.

Along with the simulation tracking, the experiments also identified the locations of the front and tail as well as the value of the coverage $c(t)^{M}$ by the IT-CTM. More exactly, the experiments first built up the IT-CTM for the testbeds, i.e. determining the time interval $\Delta t$ as well as the cell length $\Delta x$ according to the traffic data. Here, the expected length of an intermittent transmission pattern $(L=E(X)+E(Y))$ is estimated according to the average traffic condition on the entire road segment. Once $L$ is determined, $\Delta t$ and $\Delta x$ are calculated by Equation (13). Next, the experiments determined the states in each cell of the ITCTM. The expected instantaneous transmission distance $E(X)$ and the ferry distance $E(Y)$ in a cell were updated according to the traffic information in the cell in each time interval. Furthermore, we updated the 
locations of information front and tail according to inner-cell and inter-cell movement, and then updated the information coverage using the approaches proposed from Sections 3.2 to 3.6.

Table 2 below summarizes the setup of the IT-CTM under different experimental scenarios. Each scenario is conducted more than 150 runs (except scenario One-Way-PS-M-NI). Note that several experimental scenarios (see the first column in Table 2) were tested considering both one-way and twoway road segments with or without traffic interruption when a single piece or two pieces of information sequentially landing on the road segment. Moreover, it is recognized that vehicles are most likely well connected under a congested traffic flow, and then information spreads over the entire road segment very quickly. As a result, the dynamics of information spreading is not apparent. Thus, the experiments set the average flow below the capacity to avoid highly congested flow.

Table 2. IT-CTM under different experimental scenarios

\begin{tabular}{|c|c|c|c|c|c|}
\hline \hline Experiments & $\begin{array}{c}\Delta \boldsymbol{x} \\
\text { (feet) }\end{array}$ & $\begin{array}{c}\mathbf{r} \\
\text { (feet) }\end{array}$ & $\begin{array}{c}\Delta \boldsymbol{t} \\
\text { (second) }\end{array}$ & Cells & $\boldsymbol{\mu}$ and $\boldsymbol{\sigma}(\mathbf{v p h p l})$ \\
\hline One-Way-NG-S-NI & 570 & 168.17 & 6 & 4 & $1521($ N/A) \\
\hline One-Way-PS-S-NI & 990 & 203.42 & 11 & 16 & $1250(387)$ \\
\hline One-Way-PS-M-NI & 635 & 170.04 & 7 & 25 & $1500(632)$ \\
\hline Two-Way-PS-S-NI & 495 & 68.45 & 6 & 32 & $1750(431)$ \\
\hline Two-Way-PS-S-I & 610 & 86.18 & 6 & 13 & $1750(431)$ \\
\hline \multicolumn{6}{|c|}{ S: Single information; M: Multiple information; NI: no traffic interruption; I: traffic interruption } \\
NG: NGSIM data; PS: Paramics simulation data \\
\hline
\end{tabular}

\subsection{Experiment results}

The performance of the IT-CTM is evaluated from two aspects. First, this study examines the trace of the information front and tail during the course of the experiments and demonstrates if they coincide with each other. Second, this study examines the errors of the coverage rate obtained from the IT-CTM and the simulation (treated as the ground truth). Mathematically, the error is measured by the mean absolute error (MAE) in Equation (21).

$$
\operatorname{MAE}=\frac{1}{\Gamma} \sum_{t=1}^{\Gamma}\left|\left(c(t)^{M}\right)-c(t)^{S}\right|,
$$

where $\Gamma$ represents the total number of time steps in the experiments. Clearly, a smaller value of MAE indicates a more accurate estimation of $\left(c(t)^{M}\right)$ for $\left(c(t)^{S}\right)$. Note that the values of $\left(c(t)^{S}\right)$ and $\left(c(t)^{M}\right)$ as well as the absolute error (AE) are recorded at each time step.

Table 3 summarizes the average performance of the IT-CTM under different scenarios. Figure 8 shows the box-whisker plots of MAEs for some experimental scenarios. Figure 9, Figure 10, Figure 11, Figure 12, and Figure 13 present the dynamic evolvement of the information coverage, information front, and tail under respective experimental scenarios. We provide more comprehensive discussions based on these experimental results.

Table 3. The experimental results about the coverage rate under different scenarios.

\begin{tabular}{|c|c|c|c|c|c|}
\hline \hline $\begin{array}{c}\text { Experimental } \\
\text { Scenarios }\end{array}$ & \# of runs & $\begin{array}{c}\text { \# of } \\
\text { observations } \\
\text { /run }\end{array}$ & $\begin{array}{c}\text { Average MAE (for } \\
\text { information flow) }\end{array}$ & $\begin{array}{c}\text { Standard } \\
\text { Derivation } \\
\text { of MAE }\end{array}$ & Max AE \\
\hline One-Way-NG-S-NI & 157 & 21 & $3.48 \%$ & 0.06345 & $25 \%$ \\
\hline One-Way-PS-S-NI & 181 & 22 & $3.75 \%$ & 0.01284 & $18.75 \%$. \\
\hline One-Way-PS-M-NI & 1 & 18 & $2.44 \%$ & -- & $8.00 \%$ \\
\hline Two-Way-PS-S-NI & 181 & 21 & $4.72 \%$ & 0.00822 & $21.88 \%$ \\
\hline Two-Way-PS-S-I & 181 & 51 & $2.93 \%$ & 0.01422 & $23.08 \%$ \\
\hline
\end{tabular}




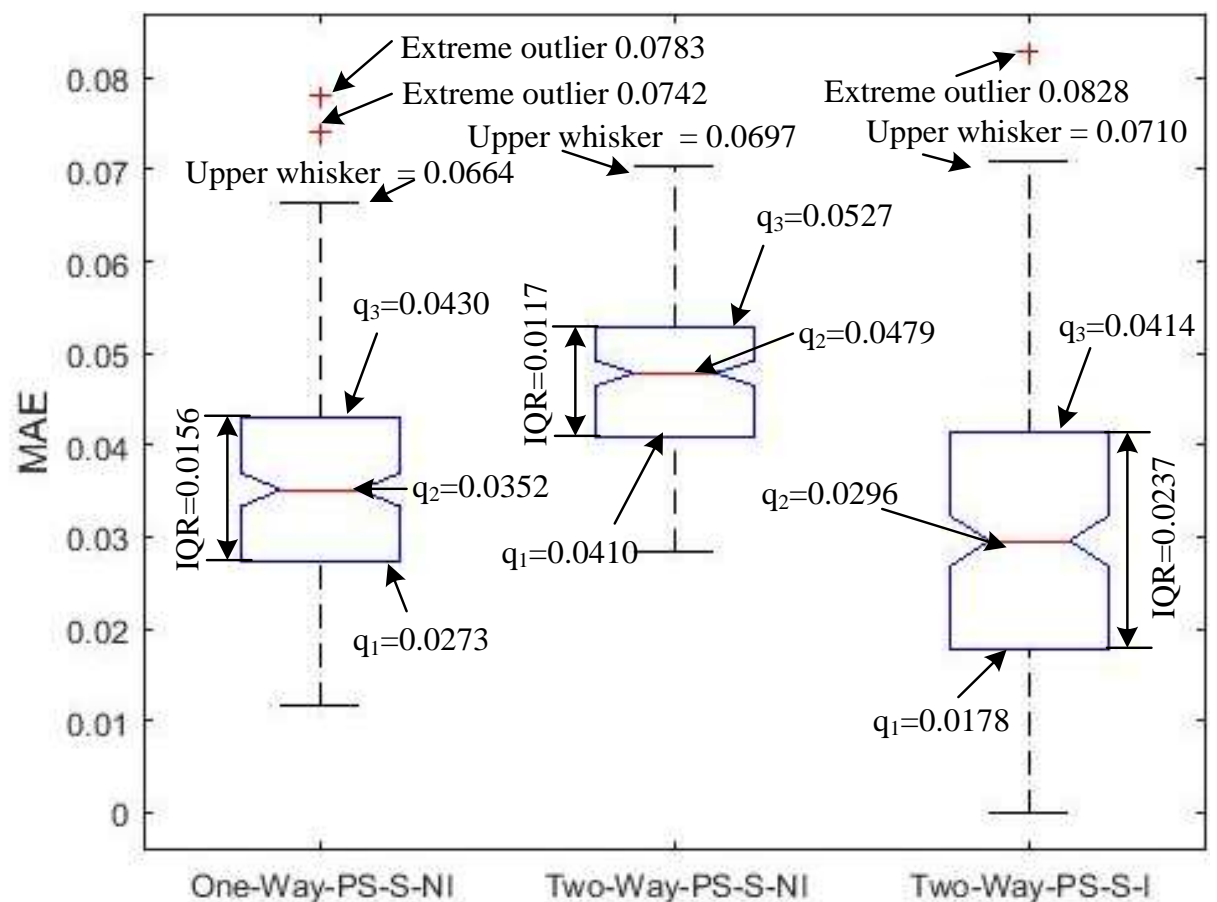

Figure 8. The box-whisker plots of three experimental scenarios

\subsubsection{Results of the experiments for the scenario of One-Way-PS-S-NI}

This study first evaluates the performance of the IT-CTM through the experiments conducted for a single piece of information landing on a one-way segment on which no significant traffic interruption occurs.

The results based on NGSIM data is presented in Table 3. It shows that the IT-CTM performs well in tracking information spreading in this scenario of experiments. More exactly, the average MAE is equal to $3.48 \%$ with a standard derivation equal to 0.06345 . The maximum $\mathrm{AE}$ is a little bit large since only 4 cells are included in this experiment. One cell difference will lead to 25\% error. With this view, this study will focus on discussing the performance of the IT-CTM on one-way road based on the experimental results using simulation data.

Figure 9 presents the experimental results using simulation data. More exactly, Figure 9 (a) shows that the information front moved forward faster than the information tail. Thus, the information front reached to the last cell of the IT-CTM (Cell 16) at the time step 1070 estimated by the IT-CTM, which is the same to what is observed from the simulation. After that, the information front stayed at Cell 16 until the experimental came to the end. However, the information tail kept on moving forward and reached to Cell 16 at the time step 1147 estimated by the IT-CTM, which is also the same as what is observed from the simulation. Hence, Figure 9 (a) indicates that the movements of information tail and front estimated by the IT-CTM presented the similar trend to the observation in the simulation, even though there are several small differences at some time steps. Accordingly, Figure 9 (b) shows that the information coverage rate increased first and then decreased to zero at the time step 1147. This is consistent with the movement of the information front and tail. Moreover, the evolvement of information coverage estimated by the ITCTM shows the similar trend to the observations from the simulation, even though it shows certain lag which results in errors occurred at some time steps.

Table 3 demonstrates the quantitative evaluation for the average performance of the IT-CTM over 181 experimental runs for scenario One-Way-PS-S-NI, while each piece of information may land on the road segment at different times. The average MAE, standard derivation of MAEs and Max AE across 181 experimental runs are equal to 3.75\%, 0.01284 and $18.75 \%$ respectively. Moreover, the box-whisker plot in Figure 8 shows that the interquartile range (IQR) is only 0.0156, thus 75\% of MAEs less than 0.0430 . Only two extreme outliers $(<8 \%$.) beyond the upper 1.5 IQR. Therefore, this study states that the IT- 
CTM can closely track the information spreading dynamics on average, even though a few relatively high errors happen occasionally.

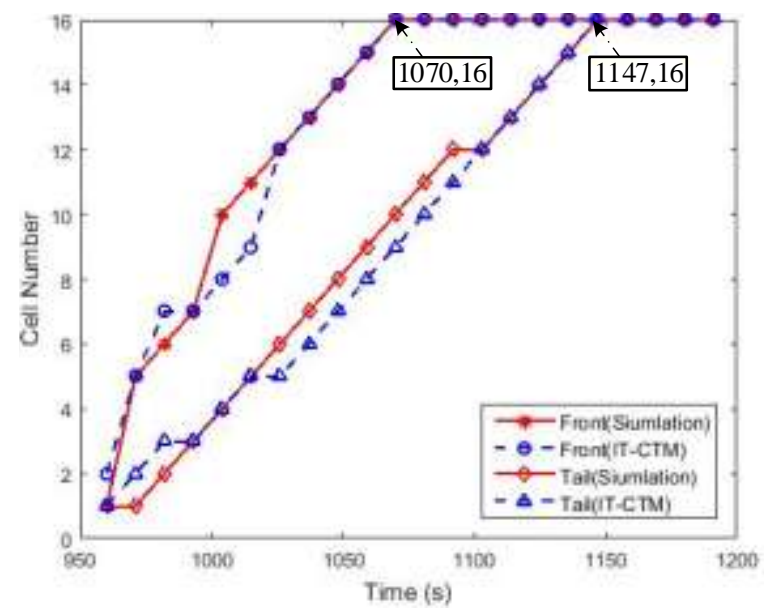

(a) Location of information front/tail

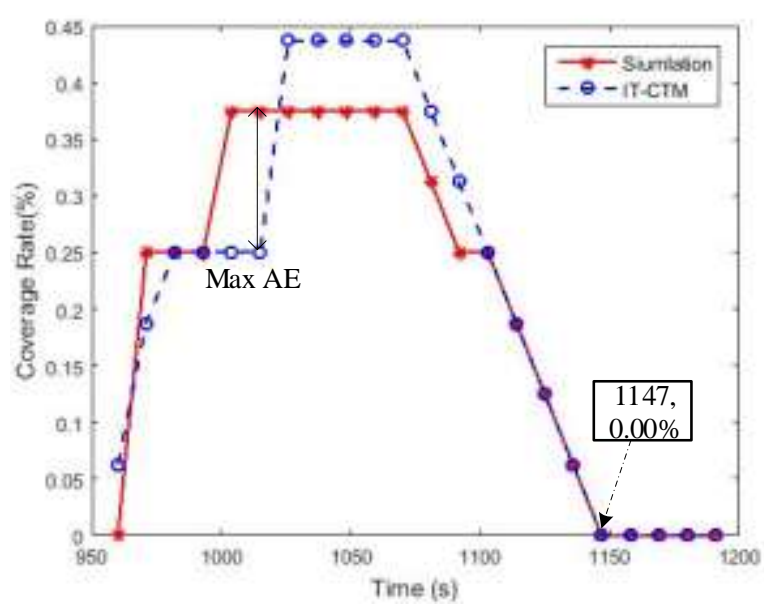

(b) Coverage rate

Figure 9. The dynamic information spreading in a run of One-Way-PS-S-NI experiment

\subsubsection{Results of the experiments for the scenario of Two-Way-PS-S-NI}

This study next evaluates the performance of the IT-CTM through the experiments conducted for a single piece of information landing on a two-way segment without significant traffic interruption. Figure 10 illustrates the dynamic spreading process of information front and tail as well as the information coverage after a single piece of information lands on at $18^{\text {th }}$ minutes (the $1080^{\text {th }}$ second).

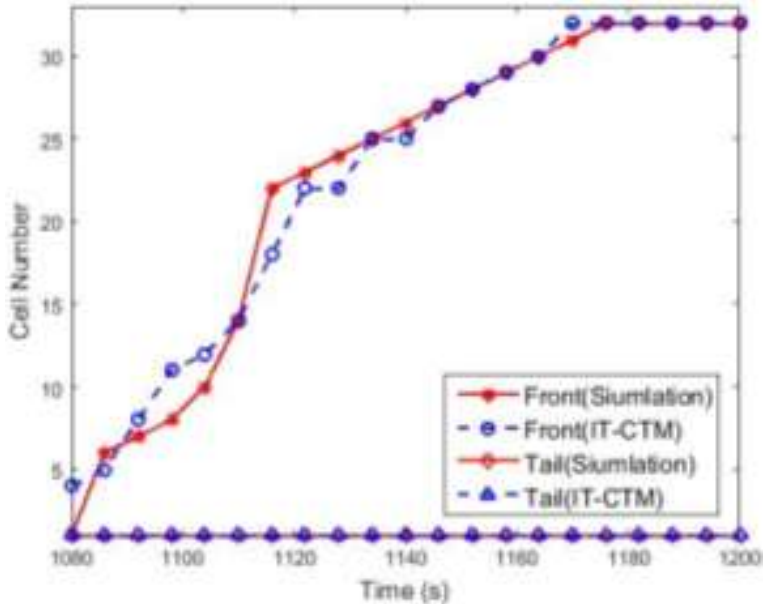

(a) Location of information front/tail

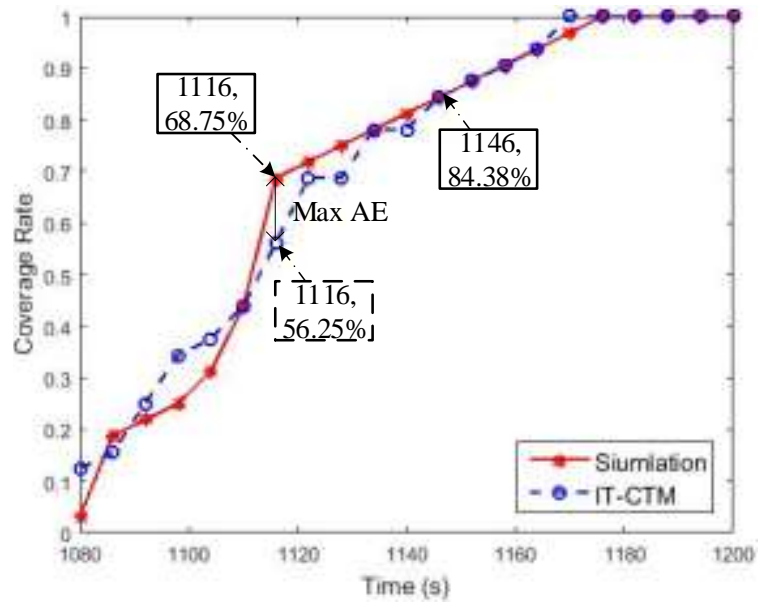

(b) Coverage rate

Figure 10. Dynamic process of information spreading in a run of Two-Way-PS-S-NI experiment

The curves in Figure 10 (a) indicate that this information front moved forward step by step until it reached the end of the road segment, while the information tail always stayed at the first cell. Figure 10 (b) indicates that the information coverage increased step by step and reached to $100 \%$ and stay there until the end of the observation. This dynamic information spreading process estimated by the IT-CTM is consistent with the observations from the simulation. Moreover, Table 3 demonstrates that the average performance of the IT-CTM for tracking information coverage over 181 experimental runs (i.e. 181 signal pieces are tracked, while each piece of information may land on the road segment at different times). The average MAE, standard derivation of MAEs and Max AE across all experimental runs are equal to 4.72\%, 0.00822 and $21.88 \%$ respectively. The box-whisker plot in Figure 8 demonstrates that the upper whisker (the maximum MAE with in 1.5IQR) is smaller than 7\%; no extreme outlier; a small IQR is equal to 
0.0117. All these statistical results indicate that the MAEs are around the average MAE and they are sufficiently small. Thus, the IT-CTM performs reasonably well.

From Figure 10 (b), this study also observed that the maximum absolute error happened around the time step 1116. After detecting the actual location and the movement of vehicles in the simulation from the time step from 1098 to 1128, this study noticed that the sudden change of the coverage rate resulted from the merge of two traffic clusters. More exactly, before the time step 1116, the space of two consecutive vehicles in each cluster was close enough to have wireless communication, but the distance between the two clusters was greater than the transmission range. However, after the time step 1116, two clusters are merged in terms of wireless communication, thus the information coverage has a sudden increase, which the IT-CTM is not able to catch. However, the average estimation error of the IT-CTM over multiple runs presents a reasonably good performance.

Next, our experiments noticed that several empty traffic cells happened in EB or in both EB and WB after the time step 1146 due to non-homogeneous traffic flow on the tested road segment. These empty traffic cells led to several boundary cases (discussed in Section 4.3). The small absolute errors (see Figure 10) after time step 1146 indicate that our approach addresses the boundary case well. Moreover, it is noticed that the information tail stayed at the first cell for a long time after the information landed on the road segment since the vehicles traveled in the opposite (such as in WB in our examples) direction always carried the information tail back to the first cell, and the information tail would only move out of the road segment if the traffic flow stops at one or both direction(s) for a while. Hence, this study conducted the experiments below.

\subsubsection{Results of the experiments for the scenario of Two-Way-PS-S-I}

This study also illustrates the capability of the IT-CTM by the experiments involving a single piece of information landing on a two-way road segment with traffic flow interruption. More exactly, a piece of information will be tracked for 5 minutes (300s), during which the traffic on the EB direction was closed for 80 seconds. In total, 181 runs of such experiments are conducted, while each piece of information may land on the road segment at different times. Note that this set of experiments shortened the road segment from 3 miles to 1.5 miles so that we can easily observe the drop of information coverage due to the traffic interruption.

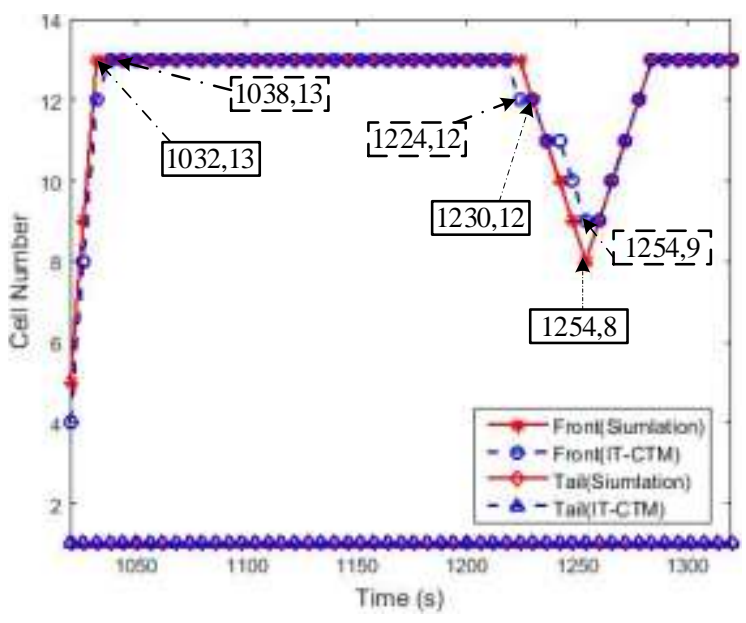

(a) Location of information front/tail

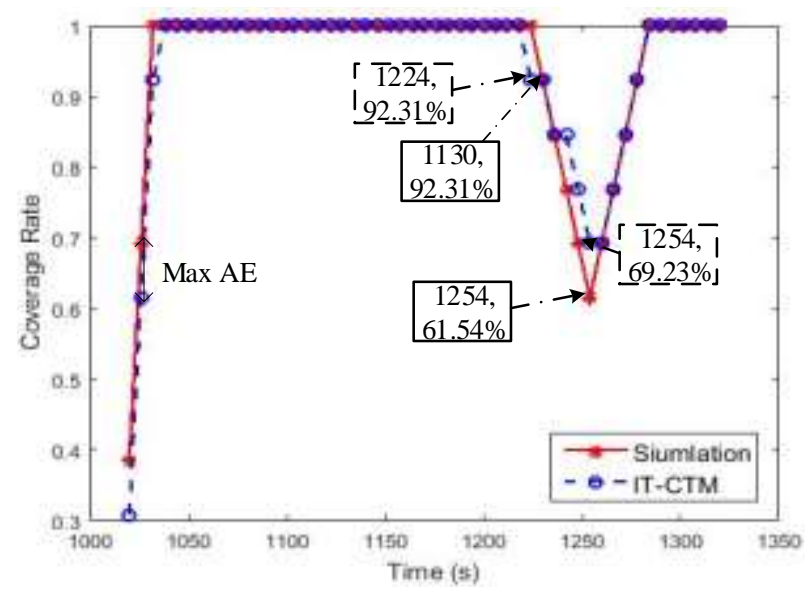

(b) Coverage rate

Figure 11. Dynamic process of information spreading in an experiment of Two-Way-PS-S-I

Figure 11 illustrates the results of an experimental run, in which a single piece of information landed on a two-way road segment at $17^{\text {th }}$ minutes (the $1020^{\text {th }}$ second) and the EB traffic was interrupted from the $1120^{\text {th }}$ second to $1200^{\text {th }}$ second. Note that we consider the EB as the forward direction of the information spreading in this experiment. Thus, the interruption of the EB traffic will remove the forward ferry in this scenario and help us to observe the effect of the backward ferry. Figure 11 (a) demonstrates 
that the information front started to move back due to the backward ferry carried by vehicles in WB around the time step 1230 in the simulation or around the time step 1224 for the IT-CTM. Accordingly, Figure 11 (b) indicates that the information coverage started to decrease around the similar time step. Furthermore, the information front in Figure 11 (a) returned back to cell 8 observed from the simulation, and cell 9 estimated by the IT-CTM. Correspondingly, the coverage in Figure 11 (b) was reduced to 61.54\% at the time step 1254 observed from the simulation, and to $69.23 \%$ at the time step 1254 . After that, the information front in Figure 11 (a) started to move forward and the information coverage started to increase again in Figure 11 (b) due to the forward transmission resulting from the recovery of the EB traffic. Overall, it is observed that the results of the IT-CTM coincide with the observations of the simulation. Table 3 provides the average performance of the IT-CTM over 181 experimental runs. The average MAE, standard derivation of MAEs and Max AE across all experiments are equal to 2.93\%, 0.01422 , and $23.08 \%$, respectively. The box-whisker plot in Figure 8 demonstrates that the IQR is equal to 0.0237 , which is sufficiently small; one extreme outlier beyond upper $1.5 \mathrm{IQR}$ is about $8 \%$. Thus, we can claim that most of MAEs are around the mean and they are sufficiently enough. Overall, the results in Figure 8, Figure 11 and Table 3 demonstrate that the IT-CTM is able to adaptively and accurately track the information spreading dynamics under a traffic flow with significant variation.

\subsubsection{The results of the experiments for the scenario of One-Way-PS-M-NI}

This study last tested the capability of the IT-CTM to track two pieces of information sequentially landing on a one-way road segment. Note that only one run of this experiment is conducted since here we focus on demonstrating the dynamics spreading and merging process of two pieces of information rather than explore the aggregated errors of coverage rate. More exactly, the first piece of information arrived at the time step 1080 and the second piece of information arrived at the time step 1115, 35-second later. We will track the spreading and merging of the two pieces of information and present the dynamic process this experiment sin Figure 12 and Figure 13.

Specifically, both the simulation and the IT-CTM curves in Figure 12 (a) indicate that the front of the second information approached to the tail of the first information gradually, and then they merged at cell 4 around the time step 1143. More exactly, the simulation results show that the merging occurred a little bit earlier than the time step estimated by the IT-CTM. Accordingly, the front of the second information vanished, but its tail was kept as the new tail of the merging information wave (see Figure 13 (a)). Similarly, the tail of the first information vanished, but its front was kept as the new front of the merged information wave (Figure 13 (b)). Figure 12 (b) demonstrates that the dynamic information coverage tracked by the IT-CTM is close to the ground truth observed from the simulation. The results in Table 3 show that both the MAE (2.44\%) and the maximum AE (8.00\%) are reasonably small in this run. Thus, this study concludes that the IT-CTM performs well under this experimental scenario.

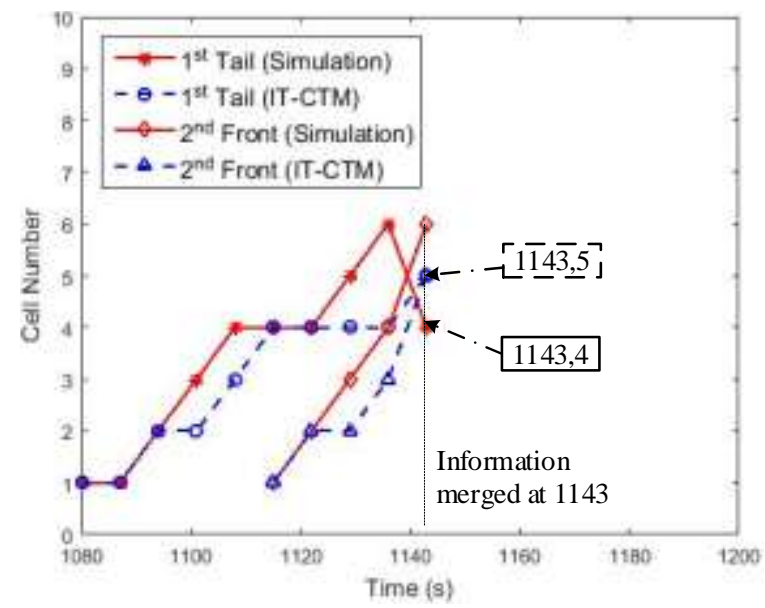

(a) Location of information front and tail

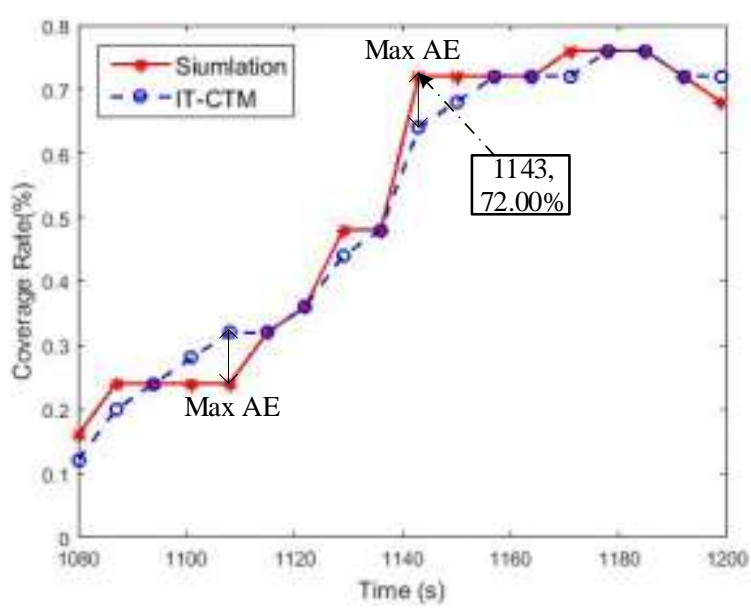

(b) Location of Coverage rate 
Figure 12. Information merging and coverage evolvement in the experiment of One-Way-PS-M-IN

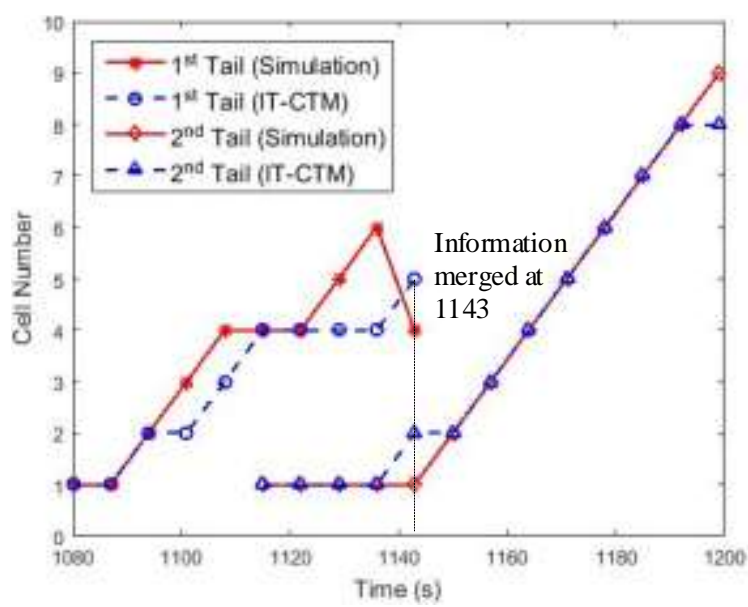

(a) Location of the tail

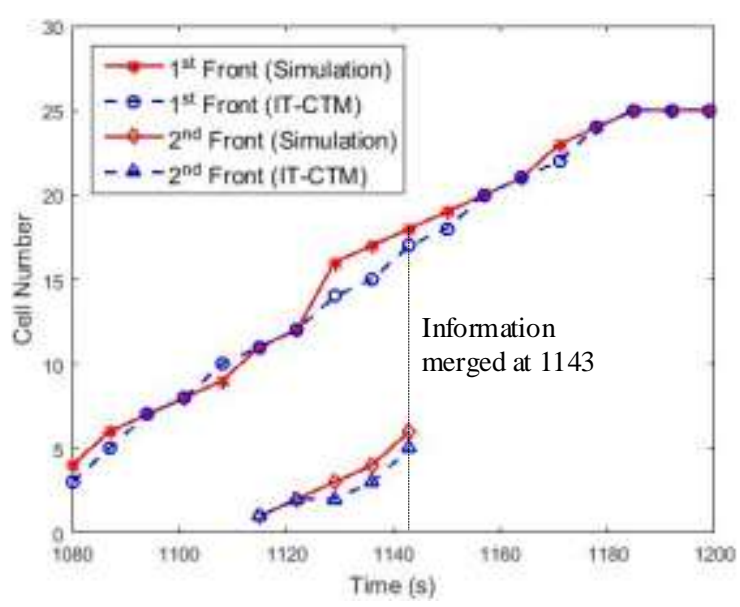

(b) Location of the front

Figure 13. Dynamic information tail/front movement in the experiment of One-Way-PS-M-IN

All the experiments on one-way, two-way road segment involving single or sequential landed information under various traffic conditions demonstrate that the IT-CTM is able to track information spreading dynamics with reasonable accuracy (the average MAE over all experiments is below 5\%.), even though a big error may occur occasionally at certain step, resulting from several approximations used in the development of the IT-CTM. For example, our experiments show that using IT-CTM to capture traffic dynamics will lead to an error within 15\%. Previous research in Du and Dao (2015) indicated that using the proposed formulations to estimate inner-cell time delay will introduce an error within 5\%. However, these errors won't have significant negative effect on the overall performance of IT-CTM.

\section{Conclusion and Future Work}

VANET plays an important role in connected vehicle systems. It possesses a great potential to improve traffic mobility, safety and sustainability in the near future. Understanding the features of the information spreading via VANET network is an essential step to successfully implement the promising applications. Having recognized this need, quite a few previous studies explored the features of information propagation in VANET on both road segment or over a traffic network, including the expected connectivity, expected instantaneous information propagating speed, distance as well as their variance, etc. However, none of them can capture the dynamic information spreading after one or multiple pieces of information land on a road segment. This research is dedicated to initiate this new line of research.

To achieve this research goal, this study developed the IT-CTM to capture the information spreading dynamics coupled with the traffic flow dynamics on a road segment. Specifically, this study considers that information spreading over a road segment along traffic flow forms an information wave with an information front and tail. An information front or tail propagates on the road segment by repeating an intermittent transmission pattern, which is consisted of an instantaneous transmission and a ferry transmission. Accordingly, the IT-CTM divides the road segment of interests into cells with a length considering the characteristics of both intermittent information transmission pattern and traffic flow. The movement of information front and tail in each cell (i.e. inner-cell information movement) is determined by three possible states (stationary, moving forward or backward), which are identified by the expected movement distance and direction of an intermittent transmission pattern. Furthermore, this study proposes the regulations for inter-cell information transmission and information spreading along a chain of cells so that the IT-CTM can dynamically capture information spreading and coverage on a road segment under 
different traffic conditions. Last, this study extends the IT-CTM approach to adapt the scenario with multiple pieces of information landing on a road segment sequentially.

The accuracy of the proposed IT-CTM approach is examined by both NGSIM field data and Paramics simulation data. Multiple experimental scenarios are conducted, including the combinations of a single or multiple piece(s) of information sequentially landing on one-way or two-way road segment with or without traffic interruption. The average mean absolute error (MAE) for tracking dynamic information coverage is less than 5\% across all experiments in this study. Therefore, this study states that the IT-CTM approach can accurately track information spreading dynamics over spatial-temporal dimensions.

Several other promising future studies are stemmed from this initial study. They include conducting more comprehensive experiments involving more realistic traffic scenarios such as traffic lights; exploring the effect of the information updating frequency or expiration time period; improving current formulations to address the lag issue; extending the current research to road network involving intersections and ramps. We propose these potential studies as our future research.

\section{Acknowledgments}

This research is partially supported by the National Science Foundation award: \#1436786. Comments from thee anonymous reviewers are also greatly appreciated.

\section{Reference}

Agarwal, A., David, S., Thomas, D. C. L., 2008. Analytical model for message propagation in delay tolerant vehicular ad hoc networks, in Proc. IEEE VTC-Spring, 3067-3071.

Alsabaan, M., Naik, K., Khalifa, T., Nayak, A., 2010. Vehicular networks for reduction of fuel consumption and $\mathrm{CO}^{2}$ emission. In Industrial Informatics (INDIN), 2010 8th IEEE International Conference, 671-676.

Al-Sultan, S., Al-Doori, M. M., Al-Bayatti, A. H., Zedan, H. (2014). A comprehensive survey on vehicular Ad Hoc network. Journal of network and computer applications, 37, 380-392.

Artimy, M. M., Phillips, W. J., Robertson, W., 2005. Connectivity with static transmission range in vehicular ad hoc networks. In Communication Networks and Services Research Conference, 2005. Proceedings of the 3rd Annual, 237-242.

Artimy, M. M., Robertson, W., Phillips, W. J., 2005. Assignment of dynamic transmission range based on estimation of vehicle density. Proceedings of the 2nd ACM international workshop on Vehicular ad hoc networks, 40-48.

Artimy, M. M., Robertson, W., Phillips, W. J., 2006. Minimum transmission range in vehicular ad hoc networks over uninterrupted highways. Intelligent Transportation Systems Conference, 2006. ITSC'06. IEEE, 1400-1405.

Bai, F., Krishnan, H., 2006. Reliability analysis of DSRC wireless communication for vehicle safety applications. In Intelligent Transportation Systems Conference, 2006. ITSC'06. IEEE, 355-362.

Bauza, R., Gozalvez, J., Sanchez-Soriano, J., 2010. Road traffic congestion detection through cooperative vehicle-to-vehicle communications. In Local Computer Networks (LCN), 2010 IEEE 35th Conference, 606-612.

Biswas, S., Tatchikou, R., Dion, F., 2006. Vehicle-to-vehicle wireless communication protocols for enhancing highway traffic safety. Communications Magazine, IEEE, 44(1), 74-82.

Burmester, M., Magkos, E., Chrissikopoulos, V., 2008. Strengthening privacy protection in VANETs. In Networking and Communications, 2008. WIMOB'08. IEEE International Conference on Wireless and Mobile Computing, 508-513.

Carey, M., Balijepalli, C., Watling, D. (2015). Extending the cell transmission model to multiple lanes and lane-changing. Networks and Spatial Economics, 15(3), 507-535.

Chen, Y., Xiang, Z., Jian, W., Jiang, W., 2009. An improved AOMDV routing protocol for V2V communication. Intelligent Vehicles Symposium, 2009 IEEE, 1115-1120.

Dar, K., Bakhouya, M., Gaber, J., Wack, M., Lorenz, P., 2010. Wireless communication technologies for ITS applications [topics in automotive networking]. Communications Magazine, IEEE, 48(5), 156-162. 
Daganzo, C. F., 1994. The cell transmission model: A dynamic representation of highway traffic consistent with the hydrodynamic theory. Transportation Research Part B: Methodological, 28(4), 269287.

Du, L., Dao, H. 2015. Information Dissemination Delay in Vehicle-to-Vehicle Communication Networks in a Traffic Stream. Intelligent Transportation Systems, IEEE Transactions on, 16(1), 66-80.

Du, L., Ukkusuri, S., 2010. The relative mobility of vehicles improves the performance of information flow in vehicle ad hoc networks," Netw. Spatial Econ., vol. 10, no. 2, pp. 209-240.

FCC, 1999. Federal Communications Commission. News Release, October 1999. FCC. Retrieved 200908-16.

Gupta, P., Kumar, P. R., 2000. The capacity of wireless networks. Information Theory, IEEE Transactions on, 46(2), 388-404.

Highway Capacity Manual. Washington, DC: Transp. Res. Board, Nat. Res. Council, 2010

Jiang, D., Taliwal, V., Meier, A., Holfelder, W., Herrtwich, R. (2006). Design of 5.9 GHz DSRC-based vehicular safety communication. IEEE Wireless Communications, 13(5), 36-43.

Jin, W. L., Recker, W. W., 2006. Instantaneous information propagation in a traffic stream through intervehicle communication. Transportation Research Part B: Methodological, 40(3), 230-250.

Jin, W. L., Recker, W. W., 2010. An analytical model of multihop connectivity of inter-vehicle communication systems. Wireless Communications, IEEE Transactions on, 9(1), 106-112.

Kim, Y., Peeta, S., He, X., 2014. An analytical model to characterize the spatiotemporal propagation of information under Vehicle-Vehicle communications, In Proc. IEEE ITS, 1142-1147.

Nekovee, M., 2006. Modeling the spread of worm epidemics in vehicular ad hoc networks In Proc. VTC, 841-845.

$\mathrm{Ng}$, M., Waller, S. T., 2010. A static network level model for the information propagation in vehicular ad hoc networks. Transportation Research Part C: Emerging Technologies, 18(3), 393-407.

Osman, O. A., Ishak, S., 2015. A network level connectivity robustness measure for connected vehicle environments. Transportation Research Part C: Emerging Technologies, 53, 48-58.

Schönhof, M., Kesting, A., Treiber, M., Helbing, D., 2006. Coupled vehicle and information flows: Message transport on a dynamic vehicle network. Physica A: Statistical Mechanics and its Applications, 363(1), 73-81.

Taleb, T., Sakhaee, E., Jamalipour, A., Hashimoto, K., Kato, N., Nemoto, Y., 2007. A stable routing protocol to support ITS services in VANET networks. Vehicular Technology, IEEE Transactions on, 56(6), 3337-3347.

Tielert, T., Killat, M., Hartenstein, H., Luz, R., Hausberger, S., Benz, T., 2010. The impact of traffic-lightto-vehicle communication on fuel consumption and emissions. In Internet of Things (IOT), 1-8.

Ukkusuri, S., Du, L., 2008. Geometric connectivity of vehicular ad hoc networks: Analytical characterization. Transportation Research Part C: Emerging Technologies, 16(5), 615-634.

Wang, X.B., 2007. Modeling the process of information relay through inter-vehicle communication. Transportation Research Part B 41 (6), 684-700

Wang, B. X., Adams, T. M., Jin, W., Meng, Q., 2010. The process of information propagation in a traffic stream with a general vehicle headway: A revisit. Transportation Research Part C: Emerging Technologies, 18(3), 367-375.

Wang, B. X., Yin, K., Zhang, Y., 2012. An exact Markov process for multihop connectivity via intervehicle communication on parallel roads. IEEE Transactions on Wireless Communications, 11(3), 865-868.

Wang, W., Liao, S. S., Li, X., Ren, J. S., 2014. The process of information propagation along a traffic stream through intervehicle communication. Intelligent Transportation Systems, IEEE Transactions on, 15(1), 345-354.

Wu, H., Fujimoto, R. M., Riley, G. F., Hunter, M., 2009. Spatial propagation of information in vehicular networks. Vehicular Technology, IEEE Transactions on, 58(1), 420-431.

Yin, K., Wang, X. B., Zhang, Y., 2013. Vehicle-to-vehicle connectivity on two parallel roadways with a general headway distribution. Transportation Research Part C: Emerging Technologies, 29, 84-96. 
Zhang, G., Wang, Y., Wei, H., Chen, Y., 2007. Examining headway distribution models with urban freeway loop event data. Transportation Research Record: Journal of the Transportation Research Board. 